\title{
Characteristic Points of Recursive Systems
}

\author{
Jason P. Bell \\ Department of Mathematics, Simon Fraser University, \\ 8888 University Dr., Burnaby, BC,V5A 1S6 \\ jpb@math.sfu.ca \\ Stanley N. Burris \\ Department of Pure Mathematics, University of Waterloo, \\ Waterloo, Ontario, N2L 3G1 \\ snburris@rogers.com \\ Karen A. Yeats \\ Department of Mathematics, Simon Fraser University, \\ 8888 University Dr., Burnaby, BC,V5A 1S6 \\ karen.yeats@math.sfu.ca
}

Submitted: May 15, 2009; Accepted: Aug 18, 2010; Published: Sep 1, 2010

Mathematics Subject Classification: 05A16

\begin{abstract}
Characteristic points have been a primary tool in the study of a generating function defined by a single recursive equation. We investigate the proper way to adapt this tool when working with multi-equation recursive systems.

Given an irreducible non-negative power series system with $m$ equations, let $\rho$ be the radius of convergence of the solution power series and let $\tau$ be the values of the solution series evaluated at $\rho$. The main results of the paper include:

(a) the set of characteristic points form an antichain in $\mathbb{R}^{m+1}$,

(b) given a characteristic point $(a, \mathbf{b})$, (i) the spectral radius of the Jacobian of $\mathbf{G}$ at $(a, \mathbf{b})$ is $\geq 1$, and (ii) it is $=1$ iff $(a, \mathbf{b})=(\rho, \boldsymbol{\tau})$,

(c) if $(\rho, \boldsymbol{\tau})$ is a characteristic point, then (i) $\rho$ is the largest $a$ for $(a, \mathbf{b})$ a characteristic point, and (ii) a characteristic point $(a, \mathbf{b})$ with $a=\rho$ is the extreme point $(\rho, \boldsymbol{\tau})$.
\end{abstract}

\section{Introduction and Preliminaries}

Recursively defined generating functions play a major role in combinatorial enumeration; see the recently published book [9] for numerous examples. The important technique of 
expressing a generating function as a product of geometric series (as well as other kinds of products) was introduced by Euler in the mid 1700s, in his study of various problems connected with the number of partitions of integers. This investigation of partition problems was continued by Sylvester and Cayley (see, for example, [5], [19]), starting in the mid 1850s. The expressions they used for partition generating functions were explicit, whereas the fundamental equation

$$
\sum_{n \geq 1} t_{n} x^{n}=x \cdot \prod_{n \geq 1}\left(1-x^{n}\right)^{-t_{n}}
$$

introduced in 1857 by Cayley [6], for rooted unlabeled trees, defined the coefficients $t_{n}$ implicitly, yielding a recursive procedure to compute the $t_{n}$. Cayley used this to recursively calculate (with some errors) the first dozen values of $t_{n}$, and later applied his method to recursively enumerate certain kinds of chemical compounds.

Let $T(x)=\sum_{n \geq 1} t_{n} x^{n}$. In 1937 Pólya (see [18]) converted (1) into

$$
T(x)=x \cdot \exp \left(\sum_{m \geq 1} T\left(x^{m}\right) / m\right)
$$

a form to which he was able to apply analytic techniques to find asymptotics for the $t_{n}$, namely he proved

$$
t_{n} \sim C \rho^{-n} n^{-3 / 2}
$$

where $\rho$ is the radius of convergence of $T(x)$, and $C$ a positive constant. ${ }^{1}$ A similar result held for the various classes of chemical compounds studied by Cayley. Although the function $T(x)$ was not expressible in terms of well-known functions, nonetheless Pólya showed how to determine $C$ and $\rho$ directly from (2). Pólya's methods were applied to nearly regular classes of trees in 1948 by Otter [17].

In 1974 Bender [1], following Pólya's ideas, formulated a general result for how to determine the radius of convergence $\rho$ of a power series $T(x)$ defined by a functional equation $F(x, y)=0$. Bender's hypotheses guaranteed that $\rho$ was positive and finite, and that $\tau:=T(\rho)$ was also finite. His method was simply to find $(\rho, \tau)$ among the solutions $(a, b)$ (called characteristic points) of the characteristic system

$$
\begin{aligned}
F(x, y) & =0 \\
\frac{\partial F}{\partial y}(x, y) & =0 .
\end{aligned}
$$

A decade later Canfield [4] found a gap in the hypotheses of Bender's formulation when there were several characteristic points. In the case of a polynomial functional equation, Canfield sketched a method to determine which of the characteristic points gives the radius of convergence of the solution $y=T(x)$.

\footnotetext{
${ }^{1}$ In [2] we found this law so ubiquitous among naturally defined classes of trees defined by a single equation that we referred to it as the universal law for rooted trees.
} 
In the late 1980s Meir and Moon [15] focused on a special case of Canfield's work, namely when $F(x, y)=0$ is of the form $y=G(x, y)$, where $G(x, y)$ is a power series with nonnegative coefficients. The interesting cases were such that setting $T(x)=G(x, T(x))$, with $T(x)$ an indeterminate power series, gave a recursive determination of the coefficients of $T(x)$. One advantage of their restricted form of recursive equation was that there could be at most one characteristic point. This formulation was adopted by Odlyzko in his 1995 survey paper [16] as well as in the recent book [9] of Flajolet and Sedgewick. These publications have focused on characteristic points in the interior of the domain of convergence of $G(x, y)$, in the context of proving that $\rho$ is a square root singularity of the solution $y=T(x)$. If $(\rho, \tau)$ is on the boundary of the domain of $G(x, y)$ then $\rho$ may not be a square-root singularity of $T(x)$.

Most areas of application actually require a recursive system of equations

$$
\left\{\begin{aligned}
y_{1} & =G_{1}\left(x, y_{1}, \ldots, y_{m}\right) \\
& \vdots \\
y_{m} & =G_{m}\left(x, y_{1}, \ldots, y_{m}\right),
\end{aligned}\right.
$$

written more briefly as $\mathbf{y}=\mathbf{G}(x, \mathbf{y})$. (A precise definition of the systems considered in this paper is given in §2.) This rich area of enumeration has been rather slow in it development. In the 1970s Berstel and Soittola (see [9] V.3) carried out a thorough analysis of enumerating the words in a regular language using recursive systems of equations that were linear in $y_{1}, \ldots, y_{m}$. However it was not until the 1990s that publications started appearing that used multi-equation non-linear systems. Following the trend with single recursion equations $y=G(x, y)$, the focus has been on systems $\mathbf{y}=\mathbf{G}(x, \mathbf{y})$ where the $G_{i}(x, \mathbf{y})$ are power series with non-negative coefficients.

In 1993 Lalley [12] considered polynomial systems in his study of random walks on free groups. In 1997 Woods [20] used one particular system to analyze the asymptotic densities of monadic second-order definable classes of trees in the class of all trees. In the same year Drmota [7] extended Lalley's results to power series systems. Lalley's and Drmota's results were for a wide range of irreducible systems, that is, systems in which each variable $y_{i}$ (eventually) depends on any variable $y_{j}$. An irreducible system of the kind they studied behaves in some ways like a single equation system, for example, the standard solution $y_{i}=T_{i}(x)$ is such that all the $T_{i}(x)$ have the same finite positive radius $\rho$, the $\tau_{i}:=T_{i}(\rho)$ are all finite, and the asymptotics for the coefficients of $T_{i}(x)$ is of the Pólya form $C_{i} \rho^{-n} n^{-3 / 2}$.

Thus, as has been the case with single equation systems, it is desirable to find the radius of convergence $\rho$ even though the solutions $T_{i}(x)$ may be fairly intractable. The natural method was to extend the definition of the characteristic system from a single equation to a system of equations, by adding the determinant of the Jacobian of the system, set equal to zero to, to the original system. The solutions of such a characteristic system will again be called characteristic points.

Under suitable conditions one can find $(\rho, \boldsymbol{\tau})$ among the characteristic points. Todate, however, the necessary study of characteristic points $(a, \mathbf{b})$ for systems, so that one can locate $(\rho, \boldsymbol{\tau})$, has been essentially non-existent. Filling this void is the goal of this 
paper. In December, 2007, we discovered, in the polynomial systems studied by Flajolet and Sedgewick, and thus in the more general systems studied by Drmota, that it was possible for there to be more than one characteristic point - this was communicated to Flajolet and appears as an example in [9] (p. 484). The main objective of this paper is to give conditions to locate $(\rho, \boldsymbol{\tau})$ among the characteristic points, if indeed $(\rho, \boldsymbol{\tau})$ is a characteristic point. A review of, and improvements to, the theory of the single equation case (see Proposition 15 and Corollary 17) are also given.

It turns out that, even if there is a characteristic point of a system $\mathbf{y}=\mathbf{G}(x, \mathbf{y})$ in the interior of the domain of $\mathbf{G}(x, \mathbf{y})$, one cannot claim that the asymptotics for the coefficients of the solutions $T_{i}(x)$ will be of the above Pólya form (see Examples 30, 31). ${ }^{2}$

We do not investigate the case when $(\rho, \boldsymbol{\tau})$ is not a characteristic point, concluding only that it must be on the boundary of the domain of $\mathbf{G}(x, \mathbf{y})$ and that the spectral radius of the Jacobian of $\mathbf{G}(x, \mathbf{y})$ at $(\rho, \boldsymbol{\tau})$ is $<1$. Note that for polynomial systems, $(\rho, \boldsymbol{\tau})$ is always a characteristic point, and in general the spectral radius condition (see Lemma 12) makes it possible to recognize when $(\rho, \boldsymbol{\tau})$ is among the characteristic points.

\subsection{Outline}

Appendix B discusses standard background and notation for power series, including a statement, Proposition 37, of the key results of Perron-Frobenius theory.

Section 2 sets up the equational systems of interest. Section 3 begins by reducing to the case where the Jacobian matrix $J_{\mathbf{G}}(x, \mathbf{y})$ has nonzero entries and then proceeds to the more interesting discussion of properties of characteristic points, including notably Proposition 11. This leads to the main result of the section, Theorem 14, followed by the single equation result, Proposition 15. Section 4 introduces an eigenvalue criterion for critical points leading to the main result of the paper, Theorem 21. Section 5 then uses the preceding results to correct an inaccuracy in the literature. The main body of the paper concludes with some open problems.

Appendix A contains a large number of examples illustrating the various possibilities and results. It is best read along side the main body of the paper.

\section{Well-conditioned systems}

The next definition gives a version of essentially well-known conditions which ensure that a system $\mathbf{y}=\mathbf{G}(x, \mathbf{y})$ as in (4) has power series solutions $y_{i}=T_{i}(x)$ of the type encountered in generating functions for classes of trees. (See Drmota [7], [8].)

\footnotetext{
${ }^{2}$ In 1997 Drmota [7] appears to claim that having a characteristic point in the interior of the domain would lead to Pólya asymptotics - however these examples show this not to be the case. In his 2009 book [8] this hypothesis is replaced with one regarding minimal characteristic points, which seems somewhat at odds with our Proposition 11, which says that the characteristic points form an antichain with the characteristic point $(a, \mathbf{b})$ of interest having the largest value of $a$ among the characteristic points. Theorem 22 of $\$ 5.1$ is a restatement of Drmota's result, to make it clear which characteristic point is of interest, namely the one (if it exists) such that the Jacobian of $\mathbf{G}(x, \mathbf{y})$ has 1 as its largest real eigenvalue.
} 
Definition 1. A system $\mathbf{y}=\mathbf{G}(x, \mathbf{y})$ is well-conditioned if it satisfies

(a) each $G_{i}(x, \mathbf{y})$ is a power series with nonnegative coefficients

(b) $\mathbf{G}(x, \mathbf{y})$ is holomorphic in a neighborhood of the origin

(c) $\mathbf{G}(0, \mathbf{y})=\mathbf{0}$

(d) for all $i, G_{i}(x, \mathbf{0}) \neq 0$

(e) $\operatorname{det}\left(I-J_{\mathbf{G}}(0, \mathbf{0})\right) \neq 0$ where $J_{\mathbf{G}}$ is the Jacobian matrix $\left(\frac{\partial G_{i}}{\partial y_{j}}\right)$

(f) the system is irreducible ${ }^{3}$

(g) for some $i, j, k, \frac{\partial^{2} G_{i}(x, \mathbf{y})}{\partial y_{j} \partial y_{k}} \neq 0$ (so the system is nonlinear in $\mathbf{y}$ ).

Remark 2. Since $\mathbf{G}(x, \mathbf{y})$ has non-negative coefficients, condition (b) is equivalent to $\left(\mathrm{b}^{\prime}\right)$ : $\mathbf{G}(x, \mathbf{y})$ converges at some positive $(a, \mathbf{b})$.

\subsection{Solutions of Well-Conditioned Systems}

The following proposition is standard.

Proposition 3. If $\mathbf{y}=\mathbf{G}(x, \mathbf{y})$ is a well-conditioned system then the following hold:

(i) There is a unique vector $\mathbf{T}(x)$ of formal power series $T_{i}(x)$ with nonnegative coefficients such that one has the formal identity

$$
\mathbf{T}(x)=\mathbf{G}(x, \mathbf{T}(x)) .
$$

(ii) Equation (5) gives a recursive procedure to find the coefficients of the $T_{i}(x)$.

(iii) Equation (5) holds for $x \in[0, \infty]$.

(iv) All $T_{i}(x)$ have the same radius of convergence $\rho \in(0, \infty)$ and all $T_{i}(x)$ converge at $\rho$, that is, $\tau_{i}:=T_{i}(\rho)<\infty$.

(v) Each $T_{i}(x)$ has a singularity at $x=\rho$.

(vi) If $(\rho, \boldsymbol{\tau})$ is in the interior of the domain of $\mathbf{G}(x, \mathbf{y})$ then

$$
\operatorname{det}\left(I-J_{\mathbf{G}}(\rho, \boldsymbol{\tau})\right)=0 \text {. }
$$

Proof. Apply Proposition 36, Pringsheim's Theorem, and the Implicit Function Theorem.

\footnotetext{
${ }^{3}$ This means the non-negative matrix $J_{\mathrm{G}}$ is irreducible.
} 
The sequence $\mathbf{T}(x)$ of power series described in Proposition 3 is the standard solution of the system, and the point $(\rho, \boldsymbol{\tau})$ is the extreme point (of the standard solution, or of the system). From (5) one has $\mathbf{T}(0)=\mathbf{0}$, so the standard solution goes through the origin. The set

$$
\operatorname{Dom}^{+}(\mathbf{G}):=\left\{(a, \mathbf{b}): a, b_{1}, \ldots, b_{m}>0 \text { and } G_{i}(a, \mathbf{b})<\infty, 1 \leq i \leq m\right\}
$$

is the positive domain of $\mathbf{G}$. For $(a, \mathbf{b}) \in \operatorname{Dom}^{+}(\mathbf{G})$ let

$$
\Lambda(a, \mathbf{b}):=\Lambda\left(J_{\mathbf{G}}(a, \mathbf{b})\right),
$$

the largest real eigenvalue of the Jacobian matrix $J_{\mathrm{G}}(a, \mathbf{b})$. Since $J_{\mathrm{G}}(a, \mathbf{b})$ is a matrix with non-negative entries, $\Lambda(a, \mathbf{b})$ is the spectral radius of $J_{\mathrm{G}}(a, \mathbf{b})$.

\subsection{Characteristic Systems, Characteristic Points}

Flajolet and Sedgewick [9] VII.6 define the characteristic system of (4) to be

$$
\left\{\begin{aligned}
y_{1} & =G_{1}\left(x, y_{1}, \ldots, y_{m}\right) \\
& \vdots \\
y_{m} & =G_{m}\left(x, y_{1}, \ldots, y_{m}\right) \\
0 & =\operatorname{det}\left(I-J_{\mathbf{G}}(x, \mathbf{y})\right) .
\end{aligned}\right.
$$

Let the positive solutions $(a, \mathbf{b}) \in \mathbb{R}^{m+1}$ to this system be called the characteristic points of the system. ${ }^{4}$ Requiring that $(\rho, \boldsymbol{\tau})$ be a characteristic point in the interior of the domain of $\mathbf{G}(x, \mathbf{y})$ has been crucial to proofs that $x=\rho$ is a square-root singularity of the $T_{i}(x)$, leading to the asymptotics $t_{i}(n) \sim C_{i} \rho^{-n} n^{-3 / 2}$ for the non-zero coefficients. There is, thus, considerable interest in finding practical computational means of estimating $\rho$.

For the case that the $G_{i}(x, \mathbf{y})$ are polynomials we know that $(\rho, \boldsymbol{\tau})$ will be among the characteristic points and in the interior of the domain of $\mathbf{G}$. However until now, even in the polynomial case, no general attempt has been made to characterize $(\rho, \boldsymbol{\tau})$ among the characteristic points of the system ${ }^{5}$ — with one exception, namely the 1-equation systems.

\section{Characteristic Points of Well-Conditioned Systems}

From now on it is assumed, unless stated otherwise, that we are working with a wellconditioned system $\Sigma: \mathbf{y}=\mathbf{G}(x, \mathbf{y})$ of $m$ equations.

\footnotetext{
${ }^{4}$ Flajolet and Sedgewick ([9] Chapter VII p. 468) only consider characteristic points in the interior of $\operatorname{Dom}^{+}(\mathbf{G})$.

${ }^{5}$ When dealing with polynomial systems in Chapter VII of [9], Flajolet and Sedgewick do not use characteristic systems - they prefer to work with the singularities, and their connections via branches, of the algebraic curves $y_{i}(x)$ defined by the system.
} 


\subsection{Making substitutions in an irreducible system}

A careful analysis of the characteristic points of $\Sigma$ is easier if $J_{\mathrm{G}}(a, \mathbf{b})$ is a positive matrix for positive points $(a, \mathbf{b})$; this is the case precisely when no entry of $J_{\mathbf{G}}(x, \mathbf{y})$ is 0 . Fortunately there is a substitution procedure to transform the original system $\Sigma$ into a well-conditioned system $\Sigma^{\star}$ with

(i) exactly the same positive solutions $(a, \mathbf{b})$, and

(ii) exactly the same set $\mathcal{C P}$ of characteristic points,

and such that for the new system $\mathbf{y}=\mathbf{G}^{\star}(x, \mathbf{y})$, the Jacobian $J_{\mathbf{G}^{\star}}(x, \mathbf{y})$ has no zero entries. Indeed, given any positive integer $n$, one can carry out the substitutions so that all $n$th partial derivatives of $\mathbf{G}(x, y)$ with respect to the $y_{i}$ are non-zero. The goal of this section is to prove these claims.

The simplest substitutions are $n$-fold iterations $\mathbf{G}^{(n)}$ of the transformation $\mathbf{G}$. These are used in [9] (see p. 492) as they suffice for aperiodic ${ }^{6}$ polynomial systems $\Sigma$. In general, however, iteration of $\mathbf{G}$ does not suffice to obtain a system $\Sigma^{\star}$ as described above - see Example 33.

Given a system $\Sigma: \mathbf{y}=\mathbf{G}(x, \mathbf{y})$, a minimal self-substitution transformation creates the system $\Sigma^{(\alpha)}: \mathbf{y}=\mathbf{G}^{(\alpha)}(x, \mathbf{y})$ by selecting $\alpha \in[0,1]$ and a pair of indices $i, j$ (possibly the same) with $\partial G_{i}(x, \mathbf{y}) / \partial y_{j} \neq 0$ and then substituting $\alpha G_{j}(x, \mathbf{y})+(1-\alpha) y_{j}$ for a single occurrence of $y_{j}$ in the power series $G_{i}$. Suppose $H\left(x, y_{0} ; \mathbf{y}\right)$ is the result of replacing the single occurrence of $y_{j}$ in $G_{i}$ by a new variable $\alpha y_{0}$. Then the system $\Sigma^{(\alpha)}$ is

$$
\Sigma^{(\alpha)}:\left\{\begin{aligned}
& y_{1}=G_{1}^{(\alpha)}(x, \mathbf{y}):=G_{1}(x, \mathbf{y}) \\
& \vdots \\
& y_{i}\left.=G_{i}^{(\alpha)}(x, \mathbf{y}):=H\left(x, \alpha G_{j}(x, \mathbf{y})+(1-\alpha) y_{j}\right) ; \mathbf{y}\right) \\
& \vdots \\
& y_{m}=G_{m}^{(\alpha)}(x, \mathbf{y}):=G_{m}(x, \mathbf{y})
\end{aligned}\right.
$$

More generally, a system $\Sigma^{\star}: \mathbf{y}=\mathbf{G}^{\star}(x, \mathbf{y})$ is a self-substitution transform of $\Sigma: \mathbf{y}=$ $\mathbf{G}(x, \mathbf{y})$ if there is a sequence $\Sigma_{0}, \Sigma_{1}, \ldots, \Sigma_{r}$ of systems such that $\Sigma=\Sigma_{0}, \Sigma^{\star}=\Sigma_{r}$, and for $0 \leq i<r$ the system $\Sigma_{i+1}$ is a minimal self-substitution transform of $\Sigma_{i}$.

Lemma 4. For $\Sigma^{(\alpha)}$ and $\Sigma^{\star}$ as described above:

(a) $\Sigma=\Sigma_{0}$.

(b) If $\Sigma$ is irreducible and $\alpha \in[0,1)$ then $\Sigma^{(\alpha)}$ is irreducible.

(c) Suppose $\Sigma$ is irreducible. Then $\Sigma^{\star}$ is irreducible iff each step $\Sigma_{i}$ is irreducible.

\footnotetext{
${ }^{6} \mathrm{~A}$ well-conditioned system $\mathbf{y}=\mathbf{G}(x, \mathbf{y})$ is aperiodic if the coefficients of each $T_{i}(x)$ are eventually positive, $\mathbf{T}(x)$ being the standard solution — see [9], p. 489.
} 
(d) Suppose $\Sigma$ is well-conditioned and $\alpha \in[0,1]$. Then $\Sigma^{(\alpha)}$ is well-conditioned iff it is irreducible. In particular $\Sigma^{(\alpha)}$ is well-conditioned if $\alpha \in[0,1)$.

(e) Suppose $\Sigma$ is well-conditioned. Then $\Sigma^{\star}$ is well-conditioned iff it is irreducible.

Proof. Straightforward.

Lemma 5. Suppose

$$
\Sigma^{\star}: \mathbf{y}=\mathbf{G}^{\star}(x, \mathbf{y})
$$

is a self-substitution transform of a well-conditioned $\Sigma: \mathbf{y}=\mathbf{G}(x, \mathbf{y})$. Then the following hold:

(a) $\mathbf{G}(x, \mathbf{y})$ and $\mathbf{G}^{\star}(x, \mathbf{y})$ have the same positive domain of convergence.

(b) $\Sigma^{\star}$ and $\Sigma$ have the same positive solutions and the same characteristic points.

(c) If $\Sigma^{\star}$ is well-conditioned then $\Sigma$ and $\Sigma^{\star}$ have the same standard solution $\mathbf{T}(\mathbf{x})$ and extreme point $(\rho, \boldsymbol{\tau})$.

(d) If $\Sigma^{\star}$ is well-conditioned then the Jacobians $J_{\mathbf{G}}(x, \mathbf{y})$ and $J_{\mathbf{G}^{\star}}(x, \mathbf{y})$ have all entries finite at the same positive points $(a, \mathbf{b})$ in the domain of $\mathbf{G}$.

Proof. It suffices to prove this for the case that $\Sigma^{\star}=\Sigma^{(\alpha)}$, a minimal self-substitution transform of $\Sigma$ as described above, namely substituting $\alpha G_{j}(x, \mathbf{y})+(1-\alpha) y_{j}$ for a single occurrence of $y_{j}$ in the power series $G_{i}(x, \mathbf{y})$. Let

$$
H\left(x, y_{0} ; \mathbf{y}\right)=A(x, \mathbf{y}) y_{0}+B(x, \mathbf{y})
$$

where $A(x, \mathbf{y})$ and $B(x, \mathbf{y})$ are power series with non-negative coefficients, and neither is 0 , be such that

$$
\begin{aligned}
G_{i}(x, \mathbf{y}) & =A(x, \mathbf{y}) y_{j}+B(x, \mathbf{y}) \\
G_{i}^{(\alpha)}(x, \mathbf{y}) & =A(x, \mathbf{y})\left(\alpha G_{j}(x, \mathbf{y})+(1-\alpha) y_{j}\right)+B(x, \mathbf{y}) .
\end{aligned}
$$

For item (a), first suppose that $(a, \mathbf{b}) \in \operatorname{Dom}^{+}(\mathbf{G})$. Then $A(a, \mathbf{b})$ and $B(a, \mathbf{b})$ are finite, so $G_{i}^{(\alpha)}(a, \mathbf{b})$ is finite. This suffices to show $(a, \mathbf{b}) \in \operatorname{Dom}^{+}\left(\mathbf{G}^{(\alpha)}\right)$ since the other $\mathbf{G}_{j}^{(\alpha)}(x, \mathbf{y})$ are the same as those in $\Sigma$. Conversely, suppose $(a, \mathbf{b}) \in \operatorname{Dom}^{+}\left(\mathbf{G}^{(\alpha)}\right)$. Again $A(a, \mathbf{b})$ and $B(a, \mathbf{b})$ are finite, so $G_{i}(a, \mathbf{b})$ is finite; and as before, the other $G_{j}(a, \mathbf{b})$ are finite. Thus $(a, \mathbf{b}) \in \operatorname{Dom}^{+}(\mathbf{G})$.

For item (b), if $i \neq j$ then clearly the two systems have the same positive solutions since $y_{j}=G_{j}(x, \mathbf{y})$ is in both systems.

If $i=j$ first note that every positive solution of $\Sigma$ is also a solution of $\Sigma^{(\alpha)}$. For the converse we have

$$
\begin{aligned}
G_{i}^{(\alpha)}(x, \mathbf{y}) & =A(x, \mathbf{y})\left(\alpha\left(A(x, \mathbf{y}) y_{i}+B(x, \mathbf{y})\right)+(1-\alpha) y_{i}\right)+B(x, \mathbf{y}) \\
& =\alpha A(x, \mathbf{y})^{2} y_{i}+\alpha A(x, \mathbf{y}) B(x, \mathbf{y})+(1-\alpha) A(x, \mathbf{y}) y_{i}+B(x, \mathbf{y})
\end{aligned}
$$


Let $(a, \mathbf{b})$ be a positive solution of $\Sigma^{(\alpha)}$. Then $(a, \mathbf{b})$ solves all equations $y_{j}=G_{j}(x, \mathbf{y})$ of $\Sigma$ where $j \neq i$ since these equations are also in $\Sigma^{(\alpha)}$. Now

$$
\begin{aligned}
b_{i} & =G_{i}^{(\alpha)}(x, \mathbf{y}) \\
& =\alpha A(a, \mathbf{b})^{2} b_{i}+\alpha A(a, \mathbf{b}) B(a, \mathbf{b})+(1-\alpha) A(a, \mathbf{b}) b_{i}+B(a, \mathbf{b}),
\end{aligned}
$$

So

$$
\left(1-\alpha A(a, \mathbf{b})^{2}-(1-\alpha) A(a, \mathbf{b})\right) b_{i}=(1+\alpha A(a, \mathbf{b})) B(a, \mathbf{b}) .
$$

Since $1+\alpha A(a, \mathbf{b})$ is positive, one can cancel to obtain

$$
b_{i}=A(a, \mathbf{b}) b_{i}+B(a, \mathbf{b})
$$

which says that $(a, \mathbf{b})$ satisfies the $i$ th equation of $\Sigma$, and thus all the equations of $\Sigma$. Consequently $\Sigma$ and $\Sigma^{(\alpha)}$ have the same positive solutions $(a, \mathbf{b})$.

To show both systems have the same characteristic points, compute

$$
\begin{aligned}
\frac{\partial G_{i}^{(\alpha)}(x, \mathbf{y})}{\partial y_{k}}= & \frac{\partial G_{i}(x, \mathbf{y})}{\partial y_{k}}+\alpha \frac{\partial A(x, \mathbf{y})}{\partial y_{k}} \cdot\left(G_{j}(x, \mathbf{y})-y_{j}\right) \\
& +\alpha A(x, \mathbf{y}) \cdot\left(\frac{\partial G_{j}(x, \mathbf{y})}{\partial y_{k}}-\delta_{j k}\right)
\end{aligned}
$$

At a positive solution $(a, \mathbf{b})$ to $\Sigma$ (hence to $\Sigma^{\star}$ ), this gives

$$
\frac{\partial G_{i}^{(\alpha)}(a, \mathbf{b})}{\partial y_{k}}=\frac{\partial G_{i}(a, \mathbf{b})}{\partial y_{k}}+\alpha A(a, \mathbf{b}) \cdot\left(\frac{\partial G_{j}(a, \mathbf{b})}{\partial y_{k}}-\delta_{j k}\right) .
$$

Thus, since $(a, \mathbf{b})$ is positive, one obtains $J_{\alpha}(a, \mathbf{b}):=I-J_{\mathbf{G}^{(\alpha)}}(a, \mathbf{b})$ from $J(a, \mathbf{b}):=$ $I-J_{\mathbf{G}}(a, \mathbf{b})$ by an elementary row operation. It follows that $\operatorname{det}(J(a, \mathbf{b}))=0$ if and only if $\operatorname{det}\left(J_{\alpha}(a, \mathbf{b})\right)=0$. Combining this with the fact that $\Sigma$ and $\Sigma^{(\alpha)}$ have the same positive solutions shows that they also have the same characteristic points.

For the next claim, item (c), note that the composition of minimal self-transforms using $\alpha \in[0,1)$ at each step preserves the well-conditioned property by Lemma 4 .

For a well-conditioned system $\Sigma$, the standard solution is the unique sequence $\mathbf{T}(x)$ of non-negative power series with $\mathbf{T}(0)=\mathbf{0}$ that solve the system. The standard solution of $\Sigma$ is clearly a solution of $\Sigma^{(\alpha)}$. Thus if $\Sigma^{(\alpha)}$ is well-conditioned then it has the same standard solution, and hence the same extreme point, as $\Sigma$, so (d) holds.

For the final item, let $(a, \mathbf{b})$ be a point in $\operatorname{Dom}^{+}(\mathbf{G})$, hence a point in $\operatorname{Dom}^{+}\left(\mathbf{G}^{(\alpha)}\right)$. $A(a, \mathbf{b})$ is finite by looking at the expression above for $\mathbf{G}_{i}(x, \mathbf{y})$. Then, since $G_{j}^{(\alpha)}(x, \mathbf{y})=$ $G_{j}(x, \mathbf{y})$ for $j \neq i,(6)$ shows that $\frac{\partial G_{i}^{(\alpha)}(a, \mathbf{b})}{\partial y_{k}}$ is finite iff $\frac{\partial G_{i}(a, \mathbf{b})}{\partial y_{k}}$ is finite, so one has item (e). 
Lemma 6. A well-conditioned system $\Sigma: \mathbf{y}=\mathbf{G}(x, \mathbf{y})$ can be transformed by a selfsubstitution into a well-conditioned system $\Sigma^{\star}: \mathbf{y}=\mathbf{G}^{\star}(x, \mathbf{y})$ such that the Jacobian matrix $J_{\mathbf{G}^{\star}}(x, \mathbf{y})$ has all entries non-zero. Indeed, given any $n>0$, one can find a $\Sigma^{\star}$ such that all nth partials of the $G_{i}^{\star}$ with respect to the $y_{j}$ are non-zero.

Proof. The goal is to show that there is a sequence $\Sigma_{0}, \ldots, \Sigma_{r}$ of minimal self-substitution transforms that go from $\Sigma$ to the desired $\Sigma^{\star}$, and such that each system $\Sigma_{i}$ is wellconditioned. The following four cases give the key steps in the proof.

CASE I: Suppose some $G_{i}$ is such that all $n$th partials are non-zero. If $G_{j}$ is dependent on $y_{i}$ (there is at least one such $j$ ) then substituting $(1 / 2) G_{i}+(1 / 2) y_{i}$ for some occurrence of $y_{i}$ in $G_{j}$ gives a well-conditioned system $\Sigma^{\prime}$ such that for $G_{i}^{\prime}=G_{i}$ and $G_{j}^{\prime}$, all $n$th partials are non-zero. Continuing in this fashion one eventually has the desired system $\Sigma^{\star}$.

CASE II: Suppose $\frac{\partial^{m n} G_{i}}{\partial y_{i}^{m n}} \neq 0$ for some $i$. This means $y_{i}^{m n}$ divides some monomial of $G_{i}$. Use the fact that for any $j \neq i$ there is a dependency path from $y_{i}$ to $y_{j}$ to convert, via self-substitutions that preserve the well-conditioned property, a product of $n$ of the $y_{i}$ in this monomial into a power series which has $y_{j}{ }^{n}$ dividing one of its monomials. By doing this for each $j \neq i$ one obtains a well-conditioned $G_{i}^{\prime}$ with

$$
\frac{\partial^{m n} G_{i}^{\prime}}{\partial y_{1}^{n} \cdots \partial y_{m}^{n}} \neq 0
$$

$\Sigma^{\prime}$ is now in Case I.

CASE III: Suppose $\frac{\partial^{2} G_{i}}{\partial y_{i}{ }^{2}} \neq 0$ for some $i$. Substituting $G_{i}$ for a suitable occurrence of $y_{i}$ in $G_{i}$ gives a well-conditioned $\Sigma^{\prime}$ where $\frac{\partial^{3} G_{i}^{\prime}}{\partial y_{i}{ }^{3}} \neq 0$. Continuing in this fashion leads to Case II.

CASE IV: Suppose $\frac{\partial^{2} G_{i}}{\partial y_{j} \partial y_{k}} \neq 0$ for some $i, j, k$. If $j \neq i$ there is a dependency path from $y_{j}$ to $y_{i}$ which shows how to make self-substitutions (that preserve the well-conditioned property) leading to $\frac{\partial^{2} G_{i}}{\partial y_{i} \partial y_{k}} \neq 0$. Likewise, if $k \neq i$ there is a dependency path from $y_{k}$ to $y_{i}$ which shows how to make self-substitutions (with each minimal step being wellconditioned) leading to $\frac{\partial^{2} G_{i}}{\partial y_{i}{ }^{2}} \neq 0$, which is Case III.

Since $\Sigma$ is non-linear in $\mathbf{y}$, for some $i, j, k$ we have

$$
\frac{\partial^{2} G_{i}}{\partial y_{i} \partial y_{k}} \neq 0
$$

Thus starting with Case IV and working back to Case I we arrive at the desired $\Sigma^{\star}$. 
Lemma 7. Let $\Sigma: \mathbf{y}=\mathbf{G}(x, \mathbf{y})$ be a well-conditioned system and let $\Sigma^{\star}: \mathbf{y}=\mathbf{G}^{\star}(x, \mathbf{y})$ be a self-substitution transform of $\Sigma$. If $(a, \mathbf{b})$ is a characteristic point of $\Sigma$, hence of $\Sigma^{\star}$, then $\Lambda(a, \mathbf{b})=1$ iff $\Lambda^{\star}(a, \mathbf{b})=1$.

Proof. Let $(a, \mathbf{b})$ be a characteristic point of $\Sigma$. It suffices to consider the case where $\Sigma^{\star}$ is obtained from $\Sigma$ by a minimal self-substitution. Let $G_{i}(x, \mathbf{y})$ depend on $y_{j}$, and let $H\left(x, y_{0} ; \mathbf{y}\right)$ be the result of replacing a single occurrence of $y_{j}$ in $G_{i}(x, \mathbf{y})$ by $y_{0}$. Then let $\Sigma^{(\alpha)}: \mathbf{y}=\mathbf{G}^{(\alpha)}(x, \mathbf{y}), \alpha \in[0,1]$, be the minimal self-substitution transform of $\Sigma$ obtained by applying the substitution $y_{0} \leftarrow \alpha G_{j}(x, \mathbf{y})+(1-\alpha) y_{j}$ to $H\left(x, y_{0} ; \mathbf{y}\right)$ to obtain

$$
\left.G_{i}^{(\alpha)}(x, \mathbf{y})=H\left(x, \alpha G_{j}(x, \mathbf{y})+(1-\alpha) y_{j}\right) ; \mathbf{y}\right) .
$$

Let $\Lambda_{\alpha}:=\Lambda_{\alpha}(a, \mathbf{b})$, the largest real eigenvalue of $J_{\mathbf{G}^{(\alpha)}}(a, \mathbf{b})$.

The only information that we need from the above construction of the $\mathbf{G}_{i}^{(\alpha)}$ is that the function $\alpha \mapsto J_{\mathbf{G}^{(\alpha)}}(a, \mathbf{b})$ is continuous on $[0,1]$, and each $J_{\mathbf{G}^{(\alpha)}}(a, \mathbf{b})$ has 1 being an eigenvalue. Since $\Lambda$ is continuous on non-negative matrices by Corollary 38, it follows that $\alpha \mapsto \Lambda_{\alpha}$ is continuous on $[0,1]$. The goal is to show that one has $\Lambda_{0}=1$ iff $\Lambda_{\alpha}=1$.

Since $(a, \mathbf{b})$ is a characteristic point of $\Sigma_{0}$ it is also a characteristic point of $\Sigma^{(\alpha)}$, by Lemma 5, for $\alpha \in[0,1]$. Thus 1 is an eigenvalue of $J_{\mathbf{G}^{(\alpha)}}(a, \mathbf{b})$ for $\alpha \in[0,1]$. Suppose $\Lambda_{0}=1$. Suppose there is a $\beta \in(0,1]$ with $\Lambda_{\beta}>1$. From the continuity of $\Lambda_{\alpha}$ there is a $\gamma \in[0, \beta)$ such that: $\Lambda_{\gamma}=1$, and $\Lambda_{\alpha}>1$ for $\alpha \in(\gamma, \beta]$.

Let $p_{\alpha}(x)$ be the characteristic polynomial of $J_{\mathbf{G}^{(\alpha)}}(a, \mathbf{b})$. From

$$
p_{\alpha}(1)=p_{\alpha}\left(\Lambda_{\alpha}\right)=0
$$

one has, for each $\alpha \in(\gamma, \beta)$, a $c_{\alpha} \in\left(1, \Lambda_{\alpha}\right)$ such that

$$
\frac{d p_{\alpha}}{d x}\left(c_{\alpha}\right)=0
$$

Since $\Lambda_{\alpha}$ is continuous on $[0,1], \lim _{\alpha \rightarrow \gamma^{+}} \Lambda_{\alpha}=\Lambda_{\gamma}=1$. This implies $\lim _{\alpha \rightarrow \gamma^{+}} c_{\alpha}=1$, and thus

$$
\frac{d p_{\gamma}}{d x}(1)=\lim _{\alpha \rightarrow \gamma^{+}} \frac{d p_{\alpha}}{d x}\left(c_{\alpha}\right)=0
$$

But from the Perron-Frobenius theory (see Proposition 37) we know that $\Lambda_{\gamma}=1$ implies that 1 is a simple root of $p_{\gamma}(x)$, giving a contradiction. Thus $\Lambda_{0}=1$ implies $\Lambda_{\alpha}=1$.

A similar proof gives the converse, that if $\Lambda_{\alpha}=1$ then $\Lambda_{0}=1$, proving the lemma.

Remark 8. In view of the last two lemmas, given a well-conditioned system $\Sigma: \mathbf{y}=$ $\mathbf{G}(x, \mathbf{y})$, when one wants to prove something about the positive solutions, the characteristic points, or whether or not $\Lambda(a, \mathbf{b})=1$ at a characteristic point $(a, \mathbf{b})$, one can, given any $n>0$, assume without loss of generality that all $n$th partials of each $G_{i}$ with respect to the $y_{j}$ are non-zero. In the rather scant literature on nonlinear systems one finds a preference for working with aperiodic systems (see, e.g., [9]), no doubt because of the simplicity of using uniform substitutions to convert such a system into one where the Jacobian of $\mathbf{G}$ has non-zero entries. With Lemmas 6 and 7 , the need for the aperiodic hypothesis is avoided. 


\subsection{Basic Properties of $(\rho, \tau)$ and $\mathcal{C P}$}

Now we turn to the question of how to find information about the extreme point $(\rho, \boldsymbol{\tau})$ of a well-conditioned system $\Sigma$ without solving the system for the standard solution $\mathbf{T}(x)$.

Lemma 9. Let $\mathbf{y}=\mathbf{G}(x, \mathbf{y})$ be a well-conditioned system with all entries of $J_{\mathbf{G}}$ non-zero.

(a) One has the formal equality

$$
\mathbf{T}^{\prime}(x)=\mathbf{G}_{x}(x, \mathbf{T}(x))+J_{\mathbf{G}}(x, \mathbf{T}(x)) \cdot \mathbf{T}^{\prime}(x),
$$

which also holds for $x \in[0, \infty]$.

(b) All $T_{i}^{\prime}(\rho)$ are finite or all $T_{i}^{\prime}(\rho)=\infty$.

(c) For all $i, j$ the following hold:

$$
\begin{aligned}
0 & <\frac{\partial G_{i}}{\partial y_{j}}(\rho, \boldsymbol{\tau}) \cdot \frac{\partial G_{j}}{\partial y_{i}}(\rho, \boldsymbol{\tau}) \leq 1 \\
0 & <\frac{\partial G_{i}}{\partial y_{j}}(\rho, \boldsymbol{\tau})<\infty \\
0 & <\frac{\partial G_{i}}{\partial y_{i}}(\rho, \boldsymbol{\tau}) \leq 1
\end{aligned}
$$

Proof. Differentiating (5) gives (7), so $\mathbf{T}^{\prime}(x)$ is a solution to the irreducible system $\mathbf{u}=$ $\mathbf{G}_{x}(x, \mathbf{T}(x))+J_{\mathbf{G}}(x, \mathbf{T}(x)) \cdot \mathbf{u}$, implying (b). For $x \in(0, \rho)$, for each $i, j,(7)$ implies

$$
T_{i}^{\prime}(x)>\frac{\partial G_{i}}{\partial y_{j}}(x, \mathbf{T}(x)) \cdot T_{j}^{\prime}(x),
$$

and thus

$$
1>\frac{\partial G_{i}}{\partial y_{j}}(x, \mathbf{T}(x)) \cdot \frac{\partial G_{j}}{\partial y_{i}}(x, \mathbf{T}(x))>0
$$

giving the inequalities in (c) since the value of $\frac{\partial G_{i}}{\partial y_{j}}(\rho, \boldsymbol{\tau})$ is the limit of $\frac{\partial G_{i}}{\partial y_{j}}(x, \mathbf{T}(x))$ as $x$ approaches $\rho$ from below.

Lemma 10. Let $\mathbf{y}=\mathbf{G}(x, \mathbf{y})$ be a well-conditioned system.

(a) If $(a, \mathbf{b}) \in \mathcal{C P}$ then $\Lambda(a, \mathbf{b}) \geq 1$.

(b) $0<\Lambda(a, \mathbf{T}(a))<1$, for $0<a<\rho$. 
Proof. For (a) note that $(a, \mathbf{b}) \in \mathcal{C P}$ implies that 1 is an eigenvalue of $J_{\mathbf{G}}(a, \mathbf{b})$, so $\Lambda(a, \mathbf{b}) \geq 1$.

(b) Given $0<a<\rho$, by the Perron-Frobenius theory of nonnegative matrices we know that there is a positive left eigenvector (a row vector) $\mathbf{v}$ belonging to $\Lambda(a, \mathbf{T}(a))$. By $(7)$

$$
\mathbf{v} \cdot \mathbf{T}^{\prime}(a)=\mathbf{v} \cdot \mathbf{G}_{x}(a, \mathbf{T}(a))+\mathbf{v} \cdot J_{\mathbf{G}}(a, \mathbf{T}(a)) \cdot \mathbf{T}^{\prime}(a),
$$

So

$$
\mathbf{v} \cdot \mathbf{T}^{\prime}(a)=\mathbf{v} \cdot \mathbf{G}_{x}(a, \mathbf{T}(a))+\Lambda(a, \mathbf{T}(a)) \mathbf{v} \cdot \mathbf{T}^{\prime}(a) .
$$

Since $\mathbf{v} \cdot \mathbf{T}^{\prime}(a)>0$ and $\mathbf{v} \cdot \mathbf{G}_{x}(a, \mathbf{T}(a))>0$ it follows that $\Lambda(a, \mathbf{T}(a))<1$.

Proposition 11. Let $\mathbf{y}=\mathbf{G}(x, \mathbf{y})$ be a well-conditioned system. Suppose $(a, \mathbf{b})$ and $(c, \mathbf{d})$ are characteristic points and $(a, \mathbf{b}) \leq(c, \mathbf{d})$. Then $(a, \mathbf{b})=(c, \mathbf{d})$. Thus the set of characteristic points of the system forms an antichain under the partial ordering $\leq$.

Proof. For the proof assume, in view of Remark 8, that all second partials of the $G_{i}$ with respect to the $y_{j}$ do not vanish. If $\mathbf{b}=\mathbf{d}$ then $\mathbf{G}(a, \mathbf{b})=\mathbf{b}=\mathbf{d}=\mathbf{G}(c, \mathbf{d})$, which forces $a=c$ by the monotonicity of each $\mathbf{G}_{i}$.

Now assume $\mathbf{b} \neq \mathbf{d}$. Since $\mathbf{b} \leq \mathbf{d}$, all entries of $\mathbf{d}-\mathbf{b}$ are non-negative. Using part of a Taylor series expansion,

$$
\mathbf{G}(c, \mathbf{d}) \geq \mathbf{G}(a, \mathbf{b})+J_{\mathbf{G}}(a, \mathbf{b})(\mathbf{d}-\mathbf{b})+\frac{1}{2}\left[\begin{array}{c}
\frac{\partial^{2} G_{1}(a, \mathbf{b})}{\partial y_{1}{ }^{2}}\left(d_{1}-b_{1}\right)^{2} \\
\vdots \\
\frac{\partial^{2} G_{m}(a, \mathbf{b})}{\partial y_{m}{ }^{2}}\left(d_{m}-b_{m}\right)^{2}
\end{array}\right]
$$

Since $\mathbf{G}(a, \mathbf{b})=\mathbf{b}$ and $\mathbf{G}(c, \mathbf{d})=\mathbf{d}$,

$$
\mathbf{d}-\mathbf{b} \geq J_{\mathbf{G}}(a, \mathbf{b})(\mathbf{d}-\mathbf{b})+\frac{1}{2}\left[\begin{array}{c}
\frac{\partial^{2} G_{1}(a, \mathbf{b})}{\partial y_{1}{ }^{2}}\left(d_{1}-b_{1}\right)^{2} \\
\vdots \\
\frac{\partial^{2} G_{m}(a, \mathbf{b})}{\partial y_{m}{ }^{2}}\left(d_{m}-b_{m}\right)^{2}
\end{array}\right] .
$$

Let $\lambda$ be the largest real eigenvalue of the positive matrix $J_{\mathbf{G}}(a, \mathbf{b})$, and let $\mathbf{v}$ be a positive left eigenvector belonging to $\lambda$. Then

$$
\begin{aligned}
& \mathbf{v}(\mathbf{d}-\mathbf{b}) \geq \mathbf{v} J_{\mathbf{G}}(a, \mathbf{b})(\mathbf{d}-\mathbf{b})+\frac{1}{2} \mathbf{v}\left[\begin{array}{c}
\frac{\partial^{2} G_{1}(a, \mathbf{b})}{\partial y_{1}{ }^{2}}\left(d_{1}-b_{1}\right)^{2} \\
\vdots \\
\frac{\partial^{2} G_{m}(a, \mathbf{b})}{\partial y_{m}{ }^{2}}\left(d_{m}-b_{m}\right)^{2}
\end{array}\right] \\
&=\lambda \mathbf{v}(\mathbf{d}-\mathbf{b})+\frac{1}{2} \mathbf{v}\left[\begin{array}{c}
\frac{\partial^{2} G_{1}(a, \mathbf{b})}{\partial y_{1}{ }^{2}}\left(d_{1}-b_{1}\right)^{2} \\
\vdots \\
\frac{\partial^{2} G_{m}(a, \mathbf{b})}{\partial y_{m}{ }^{2}}\left(d_{m}-b_{m}\right)^{2}
\end{array}\right]
\end{aligned}
$$


$\mathrm{SO}$

$$
(1-\lambda) \mathbf{v}(\mathbf{d}-\mathbf{b}) \geq \frac{1}{2} \mathbf{v}\left[\begin{array}{c}
\frac{\partial^{2} G_{1}(a, \mathbf{b})}{\partial y_{1}{ }^{2}}\left(d_{1}-b_{1}\right)^{2} \\
\vdots \\
\frac{\partial^{2} G_{m}(a, \mathbf{b})}{\partial y_{m}{ }^{2}}\left(d_{m}-b_{m}\right)^{2}
\end{array}\right]>0
$$

and this forces $\lambda<1$, contradicting Lemma 10 (a).

Lemma 12. Let $\mathbf{y}=\mathbf{G}(x, \mathbf{y})$ be a well-conditioned system.

(a) $(\rho, \boldsymbol{\tau})$ is in the domain of $J_{\mathbf{G}}(x, \mathbf{y})$, that is, all entries of the matrix $J_{\mathbf{G}}(\rho, \boldsymbol{\tau})$ are finite.

(b) If $(\rho, \boldsymbol{\tau})$ is in the interior of the domain of $\mathbf{G}(x, \mathbf{y})$ then it is a characteristic point.

(c) $0<\Lambda(\rho, \boldsymbol{\tau}) \leq 1$.

(d) $\Lambda(\rho, \boldsymbol{\tau})=1$ iff 1 is an eigenvalue of $J_{\mathbf{G}}(\rho, \boldsymbol{\tau})$ iff $(\rho, \boldsymbol{\tau}) \in \mathcal{C} \mathcal{P}$.

Proof. For item (a), first let $\Sigma^{\star}$ be a well-conditioned self-substitution transform of $\Sigma$ with all entries in $J_{\mathbf{G}^{\star}}(x, \mathbf{y})$ non-zero (see Remark 8). By Lemma 9 , all entries of $J_{\mathbf{G}^{\star}}(\rho, \boldsymbol{\tau})$ are finite. Then Lemma 5 (e) shows that all entries of $J_{\mathbf{G}}(\rho, \boldsymbol{\tau})$ are finite.

For the remainder of the proof we can assume that all entries in $J_{\mathbf{G}}$ are non-zero. For part (b) one argues just as in the case of a single equation -if $(\rho, \boldsymbol{\tau})$ is an interior point but not a characteristic point then by the implicit function theorem there would be an analytic continuation of $\mathbf{T}(x)$ at $\rho$, which is impossible.

For (c), since $\Lambda$ is a continuous nondecreasing function by Corollary 38, and since the limit of $J_{\mathbf{G}}(x, \mathbf{T}(x))$ as $x$ approaches $\rho$ from below is $J_{\mathbf{G}}(\rho, \boldsymbol{\tau})$, it follows from Lemma 10 (b) that $\Lambda(\rho, \boldsymbol{\tau}) \leq 1$.

For $(\mathrm{d})$, clearly $\Lambda(\rho, \boldsymbol{\tau})=1$ implies 1 is an eigenvalue of $J_{\mathbf{G}}(\rho, \boldsymbol{\tau})$, and this in turn implies that $(\rho, \boldsymbol{\tau}) \in \mathcal{C P}$. Now suppose that $(\rho, \boldsymbol{\tau}) \in \mathcal{C P}$. Then 1 is an eigenvalue of $J_{\mathbf{G}}(\rho, \boldsymbol{\tau})$, so $\Lambda(\rho, \boldsymbol{\tau}) \geq 1$. Thus (c) gives $\Lambda(\rho, \boldsymbol{\tau})=1$.

Lemma 13. Let $\mathbf{y}=\mathbf{G}(x, \mathbf{y})$ be a well-conditioned system. If $(a, \mathbf{b})$ is a characteristic point and $(a, \mathbf{b}) \neq(\rho, \boldsymbol{\tau})$ then either

(a) $b_{i}>\tau_{i}$ for all $i$, or

(b) $a<\rho$ and $b_{i}>T_{i}(a)$ for all $i$, and some $b_{j}>\tau_{j}$.

Proof. Condition (e) in the definition of well-conditioned ensures that each $G_{i}(x, \mathbf{y})$ depends on $x$. In view of Remark 8 assume that all second partials of each $G_{i}(x, \mathbf{y})$ with respect to the $y_{j}$ are non-zero. Suppose that (a) does not hold.

Claim 1: If some $b_{i}>\tau_{i}$ and some $b_{j} \leq \tau_{j}$ then $a<\rho$ and $T_{i}(a)<b_{i}$ for $1 \leq i \leq m$. 
WLOG assume that

$$
b_{1} \leq \tau_{1}, \ldots, b_{k} \leq \tau_{k}
$$

and

$$
b_{k+1}>\tau_{k+1}, \ldots, b_{m}>\tau_{m} .
$$

From the monotonicity and continuity of the $T_{i}$ on $[0, \rho]$ it follows that for $1 \leq i \leq k$ there exist unique $\xi_{i} \in(0, \rho]$ such that

$$
b_{i}=T_{i}\left(\xi_{i}\right) .
$$

WLOG assume that

$$
0<\xi_{1} \leq \cdots \leq \xi_{k} \leq \rho
$$

For $i \in\{1, \ldots, k\}$

$$
T_{i}\left(\xi_{1}\right) \leq T_{i}\left(\xi_{i}\right)=b_{i}
$$

and for $k+1 \leq i \leq m$

$$
T_{i}\left(\xi_{1}\right) \leq T_{i}(\rho)<b_{i} .
$$

Now suppose $\xi_{1}<a$. Then

$$
\begin{aligned}
b_{1} & =G_{1}\left(\xi_{1}, T_{1}\left(\xi_{1}\right), \ldots, T_{m}\left(\xi_{1}\right)\right) \\
& <G_{1}\left(a, b_{1}, \ldots, b_{m}\right)=b_{1},
\end{aligned}
$$

a contradiction. Thus

$$
0<a \leq \xi_{1} \leq \cdots \leq \xi_{k} \leq \rho .
$$

Using this one has, for $1 \leq i \leq k$ :

$$
\begin{aligned}
T_{i}\left(\xi_{i}\right) & =G_{i}\left(a, T_{1}\left(\xi_{1}\right), \ldots, T_{k}\left(\xi_{k}\right), b_{k+1}, \ldots, b_{m}\right) \\
& >G_{i}\left(a, T_{1}(a), \ldots, T_{k}(a), T_{k+1}(a), \ldots, T_{m}(a)\right)=T_{i}(a) .
\end{aligned}
$$

Thus for $1 \leq i \leq k$,

$$
\begin{aligned}
& 0<a<\xi_{i} \leq \rho \\
& T_{i}(a)<T_{i}\left(\xi_{i}\right)=b_{i} .
\end{aligned}
$$

Furthermore, for $k+1 \leq i \leq m$,

$$
T_{i}(a)<T_{i}(\rho)<b_{i} .
$$

Thus, in this case, for $1 \leq i \leq m$ one has $T_{i}(a)<b_{i}$.

Claim 2: If $b_{i} \leq T_{i}(\rho)$ for all $i$ then $a<\rho$ and $b_{i}=T_{i}(a)$ for all $i$.

Choose $\xi_{i} \in(0, \rho]$ such that $b_{i}=T_{i}\left(\xi_{i}\right)$. WLOG one can assume $0<\xi_{1} \leq \cdots \leq \xi_{m} \leq \rho$. If $\xi_{1}<a$ then

$$
\begin{aligned}
b_{1} & =G_{1}\left(a, T_{1}\left(\xi_{1}\right), \ldots, T_{m}\left(\xi_{m}\right)\right) \\
& >G_{1}\left(\xi_{1}, T_{1}\left(\xi_{1}\right), \ldots, T_{m}\left(\xi_{1}\right)\right) \\
& =T_{1}\left(\xi_{1}\right)=b_{1},
\end{aligned}
$$


a contradiction. Thus $a \leq \xi_{1} \leq \cdots \leq \xi_{m} \leq \rho$.

Next one has

$$
\begin{aligned}
b_{m} & =G_{m}\left(\xi_{m}, T_{1}\left(\xi_{m}\right), \ldots, T_{m}\left(\xi_{m}\right)\right) \\
& \geq G_{m}\left(a, T_{1}\left(\xi_{1}\right), \ldots, T_{m}\left(\xi_{m}\right)\right) \\
& =b_{m}
\end{aligned}
$$

so the $\geq$ step must be an equality, and this implies $\xi_{m}=a$. Thus all $\xi_{i}=a$, and then for all $i$ one has $b_{i}=T_{i}(a)$. Since $(a, \mathbf{b})=(a, \mathbf{T}(a))$ is assumed to be a different characteristic point from $(\rho, \boldsymbol{\tau})$, it follows that $a<\rho$.

Claim 3: It is not the case that $b_{i} \leq \tau_{i}$ for all $i$.

Otherwise by Claim 2 we would have $(a, \mathbf{b})=(a, \mathbf{T}(a))$ with $0<a<\rho$, and then by Lemma 10 it would follow that $(a, \mathbf{b}) \notin \mathcal{C P}$. But by assumption, $(a, \mathbf{b}) \in \mathcal{C P}$.

Theorem 14. Suppose $(\rho, \boldsymbol{\tau})$ is a characteristic point of a well-conditioned system $\mathbf{y}=$ $\mathbf{G}(x, \mathbf{y})$. Then:

(a) $\rho$ is the largest first coordinate of any characteristic point, that is

$$
\rho=\max \{a:(a, \mathbf{b}) \in \mathcal{C P}\}
$$

(b) $(\rho, \tau)$ is the only characteristic point whose first coordinate is $\rho$.

Proof. Use Proposition 11 and Lemma 13.

Turning to 1-equation systems, we have the following results.

Proposition 15. A well-conditioned 1-equation system $y=G(x, y)$ has a most one characteristic point; if there is such a point it must be the extreme point $(\rho, \tau)$ of the standard solution $T(x)$.

Proof. The characteristic system is

$$
\begin{aligned}
y & =G(x, y) \\
1 & =G_{y}(x, y) .
\end{aligned}
$$

Suppose $(a, b) \in \mathcal{C P}$ is different from $(\rho, \tau)$. Then $b>\tau$ by Lemma 13 .

CASE 1: Suppose $a>\rho$. Then $(\rho, \tau)$ is in the interior of $\operatorname{Dom}^{+}(G)$, so $(\rho, \tau) \in \mathcal{C P}$ by Lemma 12(b). But this violates the antichain condition of Proposition 11 for $\mathcal{C} \mathcal{P}$.

CASE 2: Suppose $a \leq \rho$. Then $b=G(a, b)$ and $T(a)=G(a, T(a))$ leads to $1=$ $G_{y}(a, \xi)$ for some $T(a)<\xi<b$. But $G_{y}(a, b)=1$ since $(a, b) \in \mathcal{C P}$, so again we have a contradiction by the strict monotonicity of $G_{y}(x, y)$ in $\operatorname{Dom}^{+}(G)$.

Thus the only possible $(a, b) \in \mathcal{C P}$ is $(\rho, \tau)$. 
Remark 16. Meir and Moon [15] prove that well-conditioned 1-equation systems have at most one characteristic point in the interior of $\operatorname{Dom}^{+}(G)$; and if such a point exists then it must be $(\rho, \tau)$. See also Flajolet and Sedgewick [9], Chapter VII §4.

The simple 1-equation systems $y=x A(y)$ studied by Meir and Moon appear frequently in the book [9] of Flajolet and Sedgewick. Letting $\rho_{A}$ be the radius of convergence of $A(y)$, they use the hypothesis

$$
\lim _{y \rightarrow \rho_{A^{-}}} \frac{y A^{\prime}(y)}{A(y)}>1
$$

to guarantee that $(\rho, \tau)$ is in the interior of the domain of convergence of $x A(y)$. The following corollary improves on their results by giving a precise condition for there to be a characteristic point (which must be $(\rho, \tau)$ by Proposition 15), and giving a precise condition for when $(\rho, \tau)$ is a characteristic point on the boundary [in the interior] of $\operatorname{Dom}^{+}(G)$.

Corollary 17. Suppose $y=G(x, y)$ is a well-conditioned 1-equation system with

$$
G(x, y)=x A(y),
$$

that is, $A(y)$ is a power series $\sum_{n \geq 0} a_{n} y^{n}$ with non-negative coefficients, and both $A(0)$ and $A^{\prime \prime}(y)$ are non-zero. Let $B(y)=y A^{\prime}(y)-A(y)+A(0)$. Then the characteristic system is equivalent to

$$
\begin{aligned}
B(y) & =A(0) \\
x & =\frac{y}{A(y)},
\end{aligned}
$$

and, one has

(a) $\mathcal{C P}=\varnothing$ iff $B\left(\rho_{A}\right)<A(0)$

(b) $B\left(\rho_{A}\right) \geq A(0)$ implies $\mathcal{C P}=\{(\rho, \tau)\}$

(c) $B\left(\rho_{A}\right)=A(0)$ implies $(\rho, \tau)$ is on the boundary of $\operatorname{Dom}^{+}(G)$

(d) $B\left(\rho_{A}\right)>A(0)$ implies $(\rho, \tau)$ is in the interior of $\operatorname{Dom}^{+}(G)$.

Proof. It is easy to verify the alternative form of the characteristic equations given in the corollary, and then note that

$$
B(y)=\sum_{n \geq 2}(n-1) a_{n} y^{n}
$$

is strictly increasing on $\left[0, \rho_{A}\right]$.

Remark 18. In Proposition VI.5 of [9] on simple 1-equation systems, the full wellconditioned hypothesis is not used, but instead the non-linearity condition $A^{\prime \prime}(y) \neq 0$ is replaced by the stronger condition (8). This implies $B\left(\rho_{A}\right)>A(0)$, and thus one has $(\rho, \tau)$ in the interior of $\operatorname{Dom}^{+}(\mathbf{G})$.

In the sentence following this proposition it is claimed that replacing (8) by $\rho_{A}=\infty$ gives hypotheses which imply (8). This is not correct unless one adds in the condition $A^{\prime \prime}(y) \neq 0$, that is, the correct formulation is: well-conditioned plus $\rho_{A}=\infty$ implies (8). 


\section{Eigenpoints}

The results developed so far do not give a practical way of locating $(\rho, \boldsymbol{\tau})$ for wellconditioned systems with more than one equation. Even if one is successful in finding all the characteristic points, no means has yet been formulated to determine if $(\rho, \boldsymbol{\tau})$ is among them. In this section special characteristic points called eigenpoints are shown to provide the correct analog of characteristic points when moving from 1-equation systems to multi-equation systems.

Proposition 19. Suppose $(a, \mathbf{b})$ is a characteristic point of the well-conditioned system $\mathbf{y}=\mathbf{G}(x, \mathbf{y})$. Then $\Lambda(a, \mathbf{b})=1$ iff $(a, \mathbf{b})=(\rho, \boldsymbol{\tau})$.

Proof. We can assume that no partial $\partial G_{i} / \partial y_{j}$ is zero. The direction $(\Leftarrow)$ follows from Lemma $12(\mathrm{~d})$. To prove the direction $(\Rightarrow)$ assume $(a, \mathbf{b}) \neq(\rho, \boldsymbol{\tau})$. By Lemma 13 one has two cases to consider:

(I) $a \geq \rho$ and for all $i, b_{i}>\tau_{i}$

(II) $a<\rho$ and for all $i, b_{i}>T_{i}(a)$.

For $(\mathrm{I}),(\rho, \boldsymbol{\tau})$ is in the interior of the domain of $\mathbf{G}$, so by Lemma 12 (b) it is a characteristic point. However this contradicts Proposition 11 which says the characteristic points form an antichain.

For (II), from the equations

$$
\begin{array}{r}
\mathbf{G}(a, \mathbf{b})-\mathbf{b}=\mathbf{0} \\
\mathbf{G}(a, \mathbf{T}(a))-\mathbf{T}(a)=\mathbf{0}
\end{array}
$$

one can apply a multivariate version of the mean value theorem to derive:

$$
\left(\frac{\partial G_{i}}{\partial y_{j}}\left(a, \mathbf{v}_{i j}\right)\right)(\mathbf{b}-\mathbf{T}(a))=\mathbf{b}-\mathbf{T}(a)
$$

with $\mathbf{v}_{i j}=\left(v_{i j}(1), \ldots, v_{i j}(m)\right)$ satisfying

$$
\begin{cases}v_{i j}(r)=T_{j}(a) & \text { if } r>j \\ T_{i}(a)<v_{i j}(r)<b_{i} & \text { if } r=j \\ v_{i j}(r)=b_{j} & \text { if } r<j\end{cases}
$$

Clearly (9) shows that $\lambda=1$ is an eigenvalue of $\left(\frac{\partial G_{i}}{\partial y_{j}}\left(a, \mathbf{v}_{i j}\right)\right)$, and from the properties of the $\mathbf{v}_{i j}$ we see that for all $i, j$

$$
\frac{\partial G_{i}}{\partial y_{j}}\left(a, \mathbf{v}_{i j}\right)<\frac{\partial G_{i}}{\partial y_{j}}(a, \mathbf{b})
$$

since each $\partial G_{i} / \partial y_{j}$ depends on all the variables $x, y_{1}, \ldots, y_{m}$. 
From these remarks and the monotonicity of $\Lambda$ one has

$$
1 \leq \Lambda\left(\frac{\partial G_{i}}{\partial y_{j}}\left(a, \mathbf{v}_{i j}\right)\right)<\Lambda(a, \mathbf{b}),
$$

showing that $(a, \mathbf{b}) \neq(\rho, \boldsymbol{\tau})$ implies $\Lambda(a, \mathbf{b})>1$.

Definition 20. A characteristic point $(a, \mathbf{b})$ is an eigenpoint if $\Lambda(a, \mathbf{b})=1$.

The following theorem summarizes the key results for well-conditioned systems.

Theorem 21. Let $\Sigma: \mathbf{y}=\mathbf{G}(x, \mathbf{y})$ be a well-conditioned system. Then the following hold:

(a) $(\rho, \boldsymbol{\tau}) \in \operatorname{Dom}^{+}(\mathbf{G})$

(b) If $(\rho, \boldsymbol{\tau})$ is in the interior of $\operatorname{Dom}^{+}(\mathbf{G})$ then it is an eigenpoint.

(c) The system $\Sigma$ has at most one eigenpoint.

(d) If there is an eigenpoint of $\Sigma$ then it must be $(\rho, \boldsymbol{\tau})$.

(e) If there is no eigenpoint of $\Sigma$ then $(\rho, \boldsymbol{\tau})$ lies on the boundary of $\operatorname{Dom}^{+}(\mathbf{G})$ and one has $\Lambda(\rho, \boldsymbol{\tau})<1$.

This result can be superior to Proposition 14 for computing purposes since the latter requires that one know all characteristic points of $\Sigma$ before being able to isolate the one candidate for $(\rho, \boldsymbol{\tau})$. Theorem 21 says that if one can find a characteristic point $(a, \mathbf{b})$ with $J_{\mathbf{G}}(a, \mathbf{b})$ having largest positive eigenvalue 1 , it is $(\rho, \boldsymbol{\tau})$. As with the 1-equation case, if there are no eigenpoints of $\Sigma$, then new methods are needed.

Flajolet and Sedgewick do not make use of the theory of characteristic points in their work on multi-equation systems in [9] beyond citing the work of Drmota. Instead, they consider the polynomial case in the general setting of arbitrary non-degenerate $m$-equation systems $\mathbf{P}(x, \mathbf{y})=0$ in Chap. VII.

Let $\mathcal{C}$ be the set of solution points $(a, \mathbf{b}) \in \mathbb{C}^{m+1}$ of such a system. The non-degeneracy condition implies that each $\mathcal{C}_{i}:=\left\{\left(a, b_{i}\right):(a, \mathbf{b}) \in \mathcal{C}\right\}$ is an algebraic curve. For such curves there is a simple procedure to find a finite set $X_{i}$ of points $\left(a, b_{i}\right)$ such that all singularities of $\mathcal{C}_{i}$ are in $X_{i}$.

When applying the general method of [9] to the special case of well-conditioned systems $\mathbf{y}=\mathbf{G}(x, \mathbf{y})$, to find the extreme point $(\rho, \boldsymbol{\tau})$, one can bypass the considerable work of (1) determining the branch points $\left(a, b_{i}\right)$ of the algebraic curves $\mathcal{C}_{i}$ among the points in $X_{i}$, and then (2) studying the Puiseux expansions of branches of $\mathcal{C}_{i}$ about these branch points. Instead one only needs to test the finitely many points in $\left\{(a, \mathbf{b}):\left(a, b_{i}\right) \in X_{i}\right\}$ to see which is the eigenpoint of the system - this will be $(\rho, \boldsymbol{\tau})$. 


\section{Drmota's Theorem Revisited}

In 1993 Lalley [12] proved that the solutions $y_{i}=T_{i}(x)$ to a well-conditioned polynomial system $\mathbf{y}=\mathbf{G}(x, \mathbf{y})$ would have a square-root singularity at $\rho$, and thus one had the familiar Pólya asymptotics for the coefficients. ${ }^{7}$ In 1997 [7], and again in 2009 [8], Drmota presented the first sweepingly general theorem concerning the asymptotic behavior of the coefficients of solutions of a well-conditioned system, namely the coefficients will again satisfy the same law that Pólya found to be true for several classes of trees (see [18]). However, as explained in Footnote 2, the hypotheses that Drmota has for the characteristic points of the system seem to be incorrect in the first publication, and vague in the second. ${ }^{8}$ To prove the theorem one needs to be able to show that $(\rho, \boldsymbol{\tau})$ is in the interior of the domain of $\mathbf{G}(x, \mathbf{y})$. The following subsection gives a clear statement of the hypotheses needed, along with a slightly different proof of the key induction step for the proof.

\subsection{Drmota's Theorem}

The following version is somewhat simpler than that presented by Drmota since there are no parameters.

Theorem 22. Let $\Sigma: \mathbf{y}=\mathbf{G}(x, \mathbf{y})$ be a well-conditioned system with standard solution $\mathbf{T}(x)$. Suppose $\Sigma$ has an eigenpoint $(\rho, \boldsymbol{\tau})$ in the interior of $\operatorname{Dom}^{+}(\mathbf{G})$. Then each $T_{i}(x)$ is the standard solution to a well-conditioned 1-equation system $y_{i}=\widehat{G}_{i}\left(x, y_{i}\right)$ with $\left(\rho, \tau_{i}\right)$ in the interior of $\operatorname{Dom}^{+}\left(\widehat{G}_{i}\right)$. Thus each $T_{i}(x)$ has a square-root singularity at $\rho$, and the familiar Pólya asymptotics (see, e.g., [2]) hold for the non-zero coefficients.

Proof. One only needs to consider the case that the system has at least two equations, and one can assume all second partials of the $G_{i}$ with respect to the $y_{j}$ are non-zero. The following shows that eliminating the first equation (and $y_{1}$ ) yields a well-conditioned system with one less equation which has the standard solution $\left(T_{2}(x), \ldots, T_{m}(x)\right)$ and an eigenpoint in the interior of the domain of the system.

By the Implicit Function Theorem one can solve the first equation

$$
y_{1}=\mathbf{G}_{1}(x, \mathbf{y})
$$

for $y_{1}$, say

$$
y_{1}=H_{1}\left(x, y_{2}, \ldots, y_{m}\right),
$$

where $H_{1}$ is holomorphic in a neighborhood of the origin, that is, $H_{1}(0, \mathbf{0})=0$ and

$$
H_{1}\left(x, y_{2}, \ldots, y_{m}\right)=G_{1}\left(x, H_{1}\left(x, y_{2}, \ldots, y_{m}\right), y_{2}, \ldots, y_{m}\right)
$$

\footnotetext{
${ }^{7}$ Having a polynomial system is a very strong condition since it immediately tells you that $\rho$ is a branch point, which leads to a Puiseux expansion; it is only a matter of determining the order of the branch point (which is nonetheless a nontrivial task).

${ }^{8}$ The book [9] gives a detailed study of well-conditioned polynomial systems, but only states the result for general well-conditioned systems. This statement is the 1997 version of Drmota's theorem, including the error in the hypotheses. The simplest patch is to replace the condition that 'some characteristic point $(a, \mathbf{b})$ is in the interior of the domain' with the requirement that ' $(\rho, \boldsymbol{\tau})$ is in the interior of the domain'.
} 
in a neighborhood of the origin.

Since the $T_{i}(x)$ take small values near the origin (as they are continuous functions that vanish at $x=0)$, it follows that

$$
H_{1}\left(x, T_{2}(x), \ldots, T_{m}(x)\right)=G_{1}\left(x, H_{1}\left(x, T_{2}(x), \ldots, T_{m}(x)\right), T_{2}(x), \ldots, T_{m}(x)\right)
$$

holds in a neighborhood of the origin. Also one has

$$
T_{1}(x)=G_{1}\left(x, T_{1}(x), T_{2}(x), \ldots, T_{m}(x)\right)
$$

holding in a neighborhood of the origin, so by the uniqueness of solutions in such a neighborhood, we must have

$$
T_{1}(x)=H_{1}\left(x, T_{2}(x), \ldots, T_{m}(x)\right)
$$

in a neighborhood of the origin. By Proposition 36, this equation actually holds globally for $|x| \leq \rho$; in particular $H_{1}$ converges at $\left(\rho, \tau_{2}, \ldots, \tau_{m}\right)$. By Corollary 38(a) the Jacobian $1-\frac{\partial G_{1}}{\partial y_{1}}$ of the equation $y_{1}=G_{1}(x, \mathbf{y})$ does not vanish at $(\rho, \boldsymbol{\tau})$. Thus, by the Implicit Function Theorem, $H_{1}$ is holomorphic at $\left(\rho, \tau_{2}, \ldots, \tau_{m}\right)$.

Now discarding the first equation and substituting $H_{1}\left(x, y_{2}, \ldots, y_{m}\right)$ for $y_{1}$ in the remaining equations gives a well-conditioned system of $m-1$ equations

$$
y_{i}=G_{i}^{\star}\left(x, y_{2}, \ldots, y_{m}\right),
$$

$2 \leq i \leq m$, with standard solution $\left(T_{2}(x), \ldots, T_{m}(x)\right)$ whose extreme point $\left(\rho, \tau_{2}, \ldots, \tau_{m}\right)$ is an eigenpoint, since it is a characteristic point of the system that is in the interior of $\operatorname{Dom}^{+}\left(\mathbf{G}^{\star}\right)$. Thus the elimination procedure can continue if $\mathbf{G}^{\star}$ consists of more than one equation.

The extreme point of a well-conditioned polynomial system, such as Example 32, is always a characteristic point, and, as Lalley [12] proved, the coefficients of the solutions $T_{i}(x)$ have the classical Pólya form $C_{i} \rho^{-n} n^{-3 / 2}$. Drmota [7] extended Lalley's result to well-conditioned power series systems with the extreme point in the interior of the domain of the system. A natural (and desirable) direction to consider for further research would be to drop the irreducible requirement. However, even in the polynomial case, this leads to substantial challenges, see Example 34.

\subsection{A Wealth of Examples}

In [2] we showed that single equation systems formed from a wide array of standard operators like Multiset, Cycle and Sequence led to square-root singularities and Pólya asymptotics for the coefficients. The arguments used there easily carry over to the setting of systems of equations since the conditions in that paper force the positive domain to be an open set, and this guarantees that $(\rho, \boldsymbol{\tau})$ is an interior point of the domain of the system, leading to a wealth of examples. 


\section{Some Open Problems about Characteristic Points of Well-Conditioned Systems}

Question 1. How can one locate $(\rho, \boldsymbol{\tau})$ if it is not a characteristic point?

Question 2. Is the set of characteristic points always finite?

As one can see in the examples, Appendix A, a system can have multiple characteristic points; the two equation polynomial system in Example 32 has four characteristic points. Example 35 shows that the set of real solutions to the characteristic system need not be finite. However Question 2 asks if the set of positive solutions is finite.

\section{A A Collection of Basic Examples}

The following examples explore the behavior of characteristic points of well-conditioned systems - the computational steps have been omitted. However the reader can find complete details online in the original preprint [3].

\section{A.1 Examples for 1-equation systems}

For 1-equation systems the following two examples show the three kinds of possible behavior, namely: (i) there is a characteristic point which is an interior point and thus equal to $(\rho, \tau)$, (ii) there is a characteristic point which is a boundary point and thus equal to $(\rho, \tau)$, and (iii) there is no characteristic point. If $(\rho, \tau)$ is in the interior of the domain of $G$ then $x=\rho$ is a square-root singularity of $T(x) .{ }^{9}$

Each example starts with an equation $y=G(x, y)$ where the characteristic point $(\rho, \tau)$ is in the interior of the domain of $G(x, y)$. Then the example is modified to give a system $y=G^{\star}(x, y)$ with $\left(\rho^{\star}, \tau^{\star}\right)$ on the boundary of the domain of $G^{\star}(x, y) .\left(\rho^{\star}, \tau^{\star}\right)$ is a characteristic point in Example 23 but not in Example 24.

Example 23. Let $G(x, y)=x\left(1+y^{2}\right)$. For the characteristic system

$$
\left\{\begin{array}{l}
y=x\left(1+y^{2}\right) \\
1=2 x y
\end{array}\right.
$$

of $y=G(x, y)$ one has the characteristic point $(1 / 2,1)$, an interior point of the domain of $G(x, y)$, so for the standard solution $y=S(x)$ of $y=G(x, y)$ one has $(\rho, \tau)=(1 / 2,1)$ - The established theory for such a system (see [9], Chapter VII) shows that $S(x)$ has a square-root singularity at $x=\rho$.

\footnotetext{
${ }^{9}$ The possibilities for the nature of this singularity when $(\rho, \tau)$ is on the boundary of the domain of $G$ have not been classified. Examples constructed along the lines of Proposition 27 show that one can have $2^{k}$-root singularities. Comments VI.18 and VI.19 on p. 407 of [9] state that one can have $\alpha$-root singularities, for $1<\alpha \leq 2$.
} 
Next let $G^{\star}(x, y)=S(x)\left(1+y^{2}\right) / 2$. For the characteristic system

$$
\left\{\begin{array}{l}
y=S(x)\left(1+y^{2}\right) / 2 \\
1=S(x) y
\end{array}\right.
$$

once again the characteristic point is $(1 / 2,1)$, but now it is a boundary point of the domain of $G^{*}(x, y)$. An examination of the standard solution (see Proposition 27) of $y=G^{*}(x, y)$, namely $y=T(x)=S(S(x) / 2)$, shows that it has a fourth-root singularity at $x=1 / 2$.

Example 24. Let $G(x, y)=x\left(1+2 y+2 y^{2}\right)$. The characteristic system

$$
\left\{\begin{array}{l}
y=x\left(1+2 y+2 y^{2}\right) \\
1=2 x(1+2 y)
\end{array}\right.
$$

of $y=G(x, y)$ has the characteristic point

$$
\left(\frac{\sqrt{2}-1}{2}, \frac{\sqrt{2}}{2}\right)
$$

an interior point of the domain of $G(x, y)$, so for the standard solution $y=S(x)$ of $y=G(x, y)$ one has $\rho=(\sqrt{2}-1) / 2$ and $\tau=\sqrt{2} / 2$. S $(x)$ has a square-root singularity at $x=\rho$.

Next let $G^{\star}(x, y)=x\left(1+S(x)+y+2 y^{2}\right)$. The standard solution of $y=G^{\star}(x, y)$ is again $y=S(x)$, so $\left(\rho^{*}, \tau^{*}\right)=(\rho, \tau)$. The characteristic system

$$
\left\{\begin{array}{l}
y=x\left(1+S(x)+y+2 y^{2}\right) \\
1=x(1+4 y)
\end{array}\right.
$$

of $y=G^{\star}(x, y)$ has no characteristic point since the only candidate is $(\rho, \tau)$ and

$$
\rho(1+4 \tau)=(1 / 2)(\sqrt{2}-1)(1+2 \sqrt{2}) \neq 1 .
$$

$(\rho, \tau)$ is a boundary point of the domain of $G^{*}(x, y)$ whose location is not detected by the method of characteristic points.

Remark 25. On p. 83 of their 1989 paper [15] Meir and Moon offer an interesting example of a 1-equation system without a characteristic point, namely $y=A(x) e^{y}$ where $A(x)=(1 / 6) \sum_{n} x^{n} / n^{2}$. The characteristic system is

$$
y=A(x) e^{y}, \quad 1=A(x) e^{y},
$$

so a characteristic point $(a, b)$ must have $b=1, A(a)=1 /$ e. But $1 / e$ is not in the range of $A(x)$, so there is no characteristic point. One can nonetheless easily find $(\rho, \tau)$ in this case since $(\rho, \tau)$ must lie on the boundary of the domain of $A(x) e^{y}$. Thus $\rho=1$, and then $\tau=A(1) e^{\tau}=\left(\pi^{2} / 36\right) e^{\tau}$, so $\tau \approx 0.41529$.

The paper goes on to claim that by differential equation methods one can show that the standard solution $y=S(x)$ has coefficient asymptotics $s(n) \sim C / n$. However this cannot be true since such a solution would diverge at its radius of convergence $\rho=1$ (see [2]), whereas the given equation $y=A(x) e^{y}$ is nonlinear in $y$, so the solution must converge at $\rho$.

THE ELECTRONIC JOURNAL OF COMBINATORICS 17 (2010), \#R121 


\section{A.2 1-equation framework}

This subsection gives a framework for 1-equation examples which will be useful for building the 2-equation examples in $§$ A.3.

Proposition 26. Let $A(x)$ be the standard solution of

$$
y=x\left(1+\mathfrak{a} y+\mathfrak{b} y^{2}\right)
$$

where $\mathfrak{a} \geq 0$ and $\mathfrak{b}>0$. Then the following hold:

(a)

$$
A(x)=\frac{1}{2 \mathfrak{b} x}\left((1-\mathfrak{a} x)-\sqrt{(1-\mathfrak{a} x)^{2}-4 \mathfrak{b} x^{2}}\right) .
$$

(b) $A(x)$ has non-negative coefficients.

(c) A sufficient condition for $A(x)$ to have integer coefficients is that $\mathfrak{a}$ and $\mathfrak{b}$ are integers.

(d) $A(x)$ has a positive radius of convergence $\rho_{A}$ given by

$$
\rho_{A}=\frac{1}{\mathfrak{a}+2 \sqrt{\mathfrak{b}}}
$$

(e) $\tau_{A}:=A\left(\rho_{A}\right)$ is finite and is given by

$$
\tau_{A}=\frac{1}{\sqrt{\mathfrak{b}}}
$$

(f) $\rho_{A}$ is a square-root branch point of the algebraic curve defined by (10).

(g) $\left(\rho_{A}, \tau_{A}\right)$ is the unique characteristic point of $(10)$, that is, it is the unique positive solution $(x, y)$ to

$$
\begin{aligned}
y & =x\left(1+\mathfrak{a} y+\mathfrak{b} y^{2}\right) \\
1 & =x(\mathfrak{a}+2 \mathfrak{b} y) .
\end{aligned}
$$

Proof. (Exercise.)

Proposition 27. Given $\mathfrak{a}, \mathfrak{c} \geq 0$ and $\mathfrak{b}, \mathfrak{d}>0$ let $A(x)$ be the standard solution of

$$
y=x\left(1+\mathfrak{a} y+\mathfrak{b} y^{2}\right)
$$

and let $S(x)$ be the standard solution of

$$
y=x\left(1+\mathfrak{c} y+\mathfrak{d} y^{2}\right) .
$$

Let $T(x)$ be the standard solution of

$$
y=A(x)\left(1+\mathfrak{c} y+\mathfrak{d} y^{2}\right) .
$$

Then the following hold: 
(a) $T(x)=S(A(x))$.

(b) $T(x)=\frac{1}{2 \mathfrak{d} A(x)}\left((1-\mathfrak{c} A(x))-\sqrt{(1-\mathfrak{c} A(x))^{2}-4 \mathfrak{d} A(x)^{2}}\right)$.

(c) $T(x)$ has non-negative coefficients.

(d) A sufficient condition for $T(x)$ to have integer coefficients is that $\mathfrak{a}, \mathfrak{b}, \mathfrak{c}, \mathfrak{d}$ are integers.

(e) If $\sqrt{\mathfrak{b}}=\mathfrak{c}+2 \sqrt{\mathfrak{d}}$ then

$$
\left(\rho_{T}, \tau_{T}\right)=\left(\rho_{A}, \tau_{S}\right)=\left(\frac{1}{\mathfrak{a}+2 \sqrt{\mathfrak{b}}}, \frac{1}{\sqrt{\mathfrak{d}}}\right),
$$

and $T(x)$ has a fourth-root singularity at $\rho_{T}$.

Proof. (Exercise.)

The restriction $\sqrt{\mathfrak{b}}=\mathfrak{c}+2 \sqrt{\mathfrak{d}}$ is called the critical composition condition (CCC); this is the condition needed for $T(x)=S(A(x))$ to be a critical composition (as defined by Flajolet and Sedgewick [9], p. 411).

\section{A.3 Multi-equation systems}

Proposition 28. Suppose

$$
\mathfrak{a}, \mathfrak{c}_{1} \geq 0, \quad \mathfrak{b}, \mathfrak{c}_{2}, \mathfrak{d}>0, \quad \sqrt{\mathfrak{b}}=\mathfrak{c}+2 \sqrt{\mathfrak{d}}, \quad \mathfrak{c}=\mathfrak{c}_{1}+\mathfrak{c}_{2} .
$$

Let $A(x), S(x)$, and $T(x)$ be as in Proposition 27 Then the following hold:

(a) The quadratic system

$$
(S Y S): \quad\left\{\begin{array}{l}
y_{1}=A(x)\left(1+\mathfrak{c}_{1} T(x)+\mathfrak{c}_{2} y_{2}+\mathfrak{d} y_{1}{ }^{2}\right) \\
y_{2}=A(x)\left(1+\mathfrak{c}_{1} T(x)+\mathfrak{c}_{2} y_{1}+\mathfrak{d} y_{2}^{2}\right)
\end{array}\right.
$$

is well-conditioned, and the standard solution is $y_{1}=y_{2}=T(x)$.

(b) The extreme point $(\rho, \tau, \tau)$ of $(S Y S)$ is given by

$$
(\rho, \tau, \tau)=\left(\frac{1}{\mathfrak{a}+2 \sqrt{\mathfrak{b}}}, \frac{1}{\sqrt{\mathfrak{d}}}, \frac{1}{\sqrt{\mathfrak{d}}}\right) .
$$

It is on the boundary of the domain of (SYS).

(c) $T(x)=S(A(x))$ has a fourth-root singularity at $x=\rho$. 
(d) A positive point $(x, y, y)$ is a characteristic point of (SYS) iff either

$$
(\star) \quad\left\{\begin{array}{l}
1=A(x)\left(\mathfrak{c}_{2}+2 \sqrt{\mathfrak{d}\left(1+\mathfrak{c}_{1} T(x)\right)}\right) \\
y=\frac{1-\mathfrak{c}_{2} A(x)}{2 \mathfrak{d} A(x)}
\end{array}\right.
$$

or

$$
(\star \star) \quad\left\{\begin{aligned}
1 & =A(x)\left(\mathfrak{c}_{2}+2 \sqrt{\mathfrak{c}_{2}^{2}+\mathfrak{d}\left(1+\mathfrak{c}_{1} T(x)\right)}\right) \\
y & =\frac{1+\mathfrak{c}_{2} A(x)}{2 \mathfrak{d} A(x)} .
\end{aligned}\right.
$$

(e) If $\mathfrak{c}_{1}=0$ then there are exactly two characteristic points of the form $(x, y, y)$ : the first is $(\rho, \tau, \tau)$, a boundary characteristic point obtained from $(\star)$, and the second is the unique positive solution to $(\star \star)$, an interior characteristic point. This is the only case where $(\star)$ contributes a characteristic point, namely $(\rho, \tau, \tau)$, and this is the only case where $(\rho, \tau, \tau)$ is a characteristic point.

(f) If $0<\mathfrak{c}_{1}=2 \mathfrak{c}_{2}$ then there is a unique characteristic point of the form $(x, y, y)$ : it is the unique positive solution to $(\star \star)$ and it is a boundary point different from $(\rho, \tau, \tau)$.

(g) If $0<\mathfrak{c}_{1}<2 \mathfrak{c}_{2}$ then there is a unique characteristic point of the form $(x, y, y)$ : it is the unique positive solution to $(\star \star)$ and it is an interior point that is different from $(\rho, \tau, \tau)$.

(h) If $2 \mathfrak{c}_{2}<\mathfrak{c}_{1}$ then there are no characteristic points of the form $(x, y, y)$, so again $(\rho, \tau, \tau)$ is not a characteristic point.

(i) The second characteristic point in (e) and the unique characteristic points in (f) and $(g)$ are given explicitly by

$$
\begin{aligned}
& x=\frac{\mathfrak{c}+\sqrt{\mathfrak{c}^{2}+\mathfrak{f}}}{\mathfrak{a} \mathfrak{c}+2 \mathfrak{c}^{2}+\mathfrak{f}+\mathfrak{b}+(\mathfrak{a}+2 \mathfrak{c}) \sqrt{\mathfrak{c}^{2}+\mathfrak{f}}} \\
& y=\frac{\mathfrak{c}+\mathfrak{c}_{2}+\sqrt{\mathfrak{c}^{2}+\mathfrak{f}}}{2 \mathfrak{d}}
\end{aligned}
$$

where

$$
\mathfrak{f}=-6 \mathfrak{c}_{1} \mathfrak{c}_{2}+3 \mathfrak{c}_{2}^{2}+4 \mathfrak{d}
$$

Proof. (Exercise.)

Now we look at three well-conditioned examples that show some of the varied behavior of characteristic points when one has more than one equation in the system. In the first example there are two characteristic points, both in the interior of the domain of $\mathbf{G}(x, \mathbf{y})$ and one of them is $(\rho, \boldsymbol{\tau})$. In the second example one has a characteristic point in the 
interior of the domain of $\mathbf{G}(x, \mathbf{y})$ and $(\rho, \boldsymbol{\tau})$ is a characteristic point on the boundary of the domain. In the third example one has a characteristic point in the interior of the domain of $\mathbf{G}(x, \mathbf{y})$ but $(\rho, \boldsymbol{\tau})$ is not a characteristic point. In the second and third examples, $\rho$ is not a square-root singularity of the solutions. Such examples show the need for a more subtle use of characteristic points in the pursuit of information on $(\rho, \boldsymbol{\tau})$ for multi-equation systems.

Example 29. For the system of two equations

$$
\begin{aligned}
& y_{1}=x \cdot\left(1+y_{2}+2 y_{1}^{2}\right) \\
& y_{2}=x \cdot\left(1+y_{1}+2 y_{2}^{2}\right)
\end{aligned}
$$

$a d d$

$$
\left(1-4 x y_{1}\right)\left(1-4 x y_{2}\right)-x^{2}=0
$$

to obtain the characteristic system. This is a polynomial system, so all characteristic points will be in the interior of the domain; and since $\left(\rho, \tau_{1}, \tau_{2}\right)$ is also in the interior it must be a characteristic point. Let $(a, b, c)$ be a characteristic point. By a computation we see that $b \neq c$ is impossible. Thus the characteristic points are the positive triples $(a, b, b)$ satisfying

$$
\begin{aligned}
b & =a\left(1+b+2 b^{2}\right) \\
a^{2} & =(1-4 a b)^{2} .
\end{aligned}
$$

From this the system has two characteristic points:

$$
\begin{aligned}
\left(\frac{2 \sqrt{2}-1}{7}, \frac{1}{\sqrt{2}}, \frac{1}{\sqrt{2}}\right) & \approx(0.2612,0.7071,0.7071) \\
\left(\frac{2 \sqrt{3}-1}{11}, \frac{1+\sqrt{3}}{2}, \frac{1+\sqrt{3}}{2}\right) & \approx(0.2240,1.3660,1.3660) .
\end{aligned}
$$

Now we are left with determining which of the two characteristic points gives $\left(\rho, \tau_{1}, \tau_{2}\right)$. By applying either Proposition 14 or Proposition 19, it is the first of these.

Example 30. Let $\mathfrak{a}=0, \mathfrak{b}=9, \mathfrak{c}_{1}=0, \mathfrak{c}_{2}=1$, and $\mathfrak{d}=1$. These numbers satisfy (CCC). Following the hypotheses of Proposition 28, let $A(x)$ be the standard solution to $y=x\left(1+9 y^{2}\right)$ and consider the system

$$
\begin{aligned}
& y_{1}=A(x) \cdot\left(1+y_{2}+y_{1}^{2}\right) \\
& y_{2}=A(x) \cdot\left(1+y_{1}+y_{2}^{2}\right) .
\end{aligned}
$$

Since $c_{1}=0$ there are two characteristic points of the form $(a, b, b)$. The first is the extreme point

$$
\left(\rho, \tau_{1}, \tau_{2}\right)=(1 / 6,1,1)
$$

which lies on the boundary of the domain, and the second is the interior point obtained from the formulas in Proposition 28 (i):

$$
\left(\frac{1+16 \sqrt{2}}{146}, 1+\sqrt{2}, 1+\sqrt{2}\right)
$$


Example 31. Let $\mathfrak{a}=0, \mathfrak{b}=16, \mathfrak{c}_{1}=1, \mathfrak{c}_{2}=1$, and $\mathfrak{d}=1$. These numbers satisfy (CCC). Following the hypotheses of Proposition 28, let $A(x)$ be the standard solution to $y=x\left(1+16 y^{2}\right)$, and let $T(x)$ be the standard solution to $y=A(x)\left(1+2 y+y^{2}\right)$. Consider the system

$$
\begin{aligned}
& y_{1}=A(x) \cdot\left(1+T(x)+y_{2}+y_{1}^{2}\right) \\
& y_{2}=A(x) \cdot\left(1+T(x)+y_{1}+y_{2}^{2}\right) .
\end{aligned}
$$

Since $0<c_{1}<2 c_{2}$, the extreme point

$$
\left(\rho, \tau_{1}, \tau_{2}\right)=(1 / 8,1,1)
$$

is not a characteristic point, but there is a characteristic point of the form $(a, b, b)$ in the interior of the domain of $\mathbf{G}$ given by the formulas of Proposition 28:

$$
(a, b, b)=\left(\frac{30+17 \sqrt{5}}{545}, \frac{3+\sqrt{5}}{2}, \frac{3+\sqrt{5}}{2}\right) .
$$

\section{A.4 Other examples}

The next example shows some characteristic points which are not of the form $(x, y, y)$

Example 32. The well-conditioned polynomial system

$$
\begin{aligned}
& y_{1}=G_{1}\left(x, y_{1}, y_{2}\right):=x\left(1+2 y_{1}^{3}+2 x^{3} y_{1}^{3} y_{2}\right) \\
& y_{2}=G_{2}\left(x, y_{1}, y_{2}\right):=x\left(1+x^{3} y_{2}+2 y_{1}^{3} y_{2}^{2}\right)
\end{aligned}
$$

has four characteristic points which, to 6 places of accuracy are:

$(0.1818598,1.556545,0.3647603)$

$(0.2640956,1.210710,0.5353688)$

$(0.3867644,0.6661246,3.834789)$

$(0.4153198,0.6217456,0.4743552)$

One sees that these four points form an antichain, as required by Proposition 11. The extreme point $\left(\rho, \tau_{1}, \tau_{2}\right)$ of a polynomial system is a characteristic point. By Proposition 14 it must be the last one since it has the largest $x$-value, assuming one has found all characteristic roots of this system. If one is not sure that there are only four characteristic points then, by Theorem 21, it suffices to verify that the indicated characteristic point is an eigenpoint.

This example demonstrates that iteration is not sufficient to obtain a new systems $\Sigma^{\star}$ such that the Jacobian matrix $J_{\mathbf{G}^{\star}}(x, \mathbf{y})$ has non-zero entries. 
Example 33. Consider the irreducible system $\mathbf{y}=\mathbf{G}(x, \mathbf{y})$ of 4 equations:

$$
\Sigma=\left\{\begin{array}{l}
y_{1}=G_{1}\left(x, y_{1}, \ldots, y_{4}\right):=x\left(1+y_{2}^{2}+y_{4}^{2}\right) \\
y_{2}=G_{2}\left(x, y_{1}, \ldots, y_{4}\right):=x\left(1+y_{1}^{2}+y_{3}^{2}\right) \\
y_{3}=G_{3}\left(x, y_{1}, \ldots, y_{4}\right):=x\left(1+y_{4}^{2}\right) \\
y_{4}=G_{4}\left(x, y_{1}, \ldots, y_{4}\right):=x\left(1+y_{1}^{2}\right) .
\end{array}\right.
$$

Let $M=J_{\mathbf{G}^{(n)}}$. Then it is easy to check that $M_{11} \neq 0$ iff $n$ is odd, and $M_{12} \neq 0$ iff $n$ is even. Thus for $n \geq 1, J_{\mathbf{G}^{(n)}}(x, \mathbf{y})$ has entries which are 0 .

One can transform $\Sigma$ into a system $\Sigma^{\star}$ where the Jacobian of $\mathbf{G}^{\star}$ has all entries nonzero by doing selective substitutions. For example, in the first equation of $\Sigma$ replace one of the two $y_{2}$ 's by $G_{2}(x, \mathbf{y})$, giving the system

$$
\left\{\begin{array}{l}
y_{1}=x\left(1+y_{2} G_{2}(x, \mathbf{y})+y_{4}^{2}\right) \\
y_{2}=x\left(1+y_{1}^{2}+y_{3}^{2}\right) \\
y_{3}=x\left(1+y_{4}^{2}\right) \\
y_{4}=x\left(1+y_{1}^{2}\right)
\end{array}\right.
$$

The first equation in this system is such that the right hand side depends on all 4 of the $y_{i}$. Continuing in this manner one obtains a system in which every $G_{i}(x, \mathbf{y})$ depends on each of $y_{1}, \ldots, y_{4}$.

This example shows complications which can arise with reducible systems.

Example 34. Consider the reducible polynomial system

$$
\begin{aligned}
& y_{1}=y_{3} \cdot\left(1+y_{2}+y_{1}^{2}\right) \\
& y_{2}=y_{3} \cdot\left(1+y_{1}+y_{2}^{2}\right) \\
& y_{3}=x \cdot\left(1+9 y_{3}{ }^{2}\right) .
\end{aligned}
$$

Let the third equation have the standard solution $y_{3}=A(x)$. One then sees that this example is really just an alternate presentation of Example 30 where the solutions for $y_{1}$ and $y_{2}$ have a fourth-root singularity at their radius of convergence.

This final example shows that there can be infinitely many real solutions to a characteristic system, in contrast to what has been observed so far for characteristic points, see Question 2.

Example 35. For the characteristic system (belonging to a 2-equation system)

$$
\begin{cases}y_{1}-x \cdot\left(1+y_{1}+y_{1} y_{2}\right) & =0 \\ y_{2}-x \cdot\left(1+y_{2}+y_{1} y_{2}\right) & =0 \\ (x-1) \cdot\left(x+x y_{1}+x y_{2}-1\right) & =0\end{cases}
$$

the real solutions include the infinite curve

$$
\left\{\left(x, y_{1}, y_{2}\right): x=1, y_{1} y_{2}=-1\right\}
$$




\section{B Background Material}

\section{B.1 The extended nonnegative real numbers}

Extend the usual operations on $[0, \infty)$ to $[0, \infty]$ in the obvious way as follows:

$$
\begin{aligned}
c+\infty & =\infty \text { for } c \in[0, \infty] \\
c \cdot \infty & =\infty \text { for } c \in(0, \infty] \\
\sum_{n} c_{n} & = \begin{cases}\text { the usual infinite sum } & \text { if all } c_{n} \in[0, \infty) \\
\infty & \text { if some } c_{n}=\infty\end{cases}
\end{aligned}
$$

Here the usual infinite sum is $\infty$ if the series diverges. Note that $0 \cdot \infty$ is left undefined since it is indeterminate.

\section{B.2 Formal power series in several variables}

This section gives the essential definitions that lay the foundations for working with formal power series in several variables. The standard number systems are:

the set $\mathbb{N}=\{0,1, \ldots\}$ of nonnegative integers, the set $\mathbb{Q}$ of rational numbers, the set $\mathbb{R}$ of real numbers, and the set $\mathbb{C}$ of complex numbers.

For the linearly ordered set $\mathbb{R}$ of real numbers one has the posets of real-valued functions on $X$, where the partial ordering is given by $f \leq g$ if $f(x) \leq g(x)$ for all $x \in X$. Familiar examples are:

(a) $n$-vectors $\mathbf{v}=\left(v_{1}, \ldots, v_{n}\right)$, by setting $X=\{1, \ldots, n\}$

(b) $m \times n$-matrices $M$, by setting $X=\{1, \ldots, m\} \times\{1, \ldots, n\}$

(c) formal power series in $k$-variables $A\left(x_{1}, \ldots, x_{k}\right)$ by setting $X=\mathbb{N}^{k}$. In this case a function $a$ from $\mathbb{N}^{k}$ to $\mathbb{R}$ provides the coefficients, and one writes

$$
A(\mathbf{x}):=\sum_{\mathbf{i} \in \mathbb{N}^{k}} a(\mathbf{i}) \mathbf{x}^{\mathbf{i}}
$$

A matrix (or vector) $M$ of real numbers is non-negative (written $M \geq 0$ ) if each entry is non-negative, and positive (written $M>0$ ) if each entry is positive. A power series $A(\mathbf{x})$ is non-negative (written $A(\mathbf{x}) \geq 0$ ) if each coefficient is non-negative. 


\section{B.2.1 Composition of formal power series}

For power series $A\left(w_{1}, \ldots, w_{m}\right)$ and $B_{\ell}(\mathbf{x}), 1 \leq \ell \leq m$, where the constant term of each $B_{\ell}$ is zero, that is, $b_{\ell}(\mathbf{0})=0$, define the formal composition

$$
C(\mathbf{x}):=A\left(B_{1}(\mathbf{x}), \ldots, B_{m}(\mathbf{x})\right)
$$

by defining the coefficient function as follows:

$$
c(\mathbf{i}):=\sum_{\mathbf{j} \geq \mathbf{0}}\left[\mathbf{x}^{\mathbf{i}}\right] a(\mathbf{j}) \cdot B_{1}(\mathbf{x})^{j_{1}} \cdots B_{m}(\mathbf{x})^{j_{m}}
$$

Requiring that the constant term of the $B_{\ell}(\mathbf{x})$ be 0 guarantees that for each $\mathbf{i}$ only finitely many terms in this sum are nonzero. Consequently $C(\mathbf{x})$ is indeed a formal power series.

\section{B.2.2 The function defined by a formal power series}

A power series $A(\mathbf{x})$ in $k$ variables defines a partial function, also denoted $A(\mathbf{x})$, on $\mathbb{R}^{k}$ $\left(\right.$ or $\mathbb{C}^{k}$ ) by setting

$$
A(\mathbf{c}):=\sum_{n \geq 0} \sum_{i_{1}+\cdots+i_{k}=n} a(\mathbf{i}) \mathbf{c}^{\mathbf{i}} \quad\left(\mathbf{c} \in \mathbb{R}^{k}\right)
$$

whenever the sum converges.

For $A(\mathbf{x})$ a nonnegative power series in $k$ variables, and for $\mathbf{c} \in[0, \infty]^{k}, A(\mathbf{c})=\infty$ if the series (11) diverges, that is, if

$$
\lim _{n \rightarrow \infty} \sum_{j \leq n} \sum_{i_{1}+\cdots+i_{k}=j} a(\mathbf{i}) \mathbf{c}^{\mathbf{i}}=\infty .
$$

A nonnegative power series $A(\mathbf{x})$ in $k$ variables defines a left-continuous function from $[0, \infty]^{k}$ to $[0, \infty]$ and is monotone nondecreasing in each variable on $[0, \infty]^{k}$.

\section{B.2.3 The derivatives of a formal power series}

Derivatives of [nonnegative] formal power series give [nonnegative] formal power series:

$$
\frac{\partial A(\mathbf{x})}{\partial x_{j}}:=\sum_{\mathbf{i} \geq \mathbf{0}} i_{j} a(\mathbf{i}) x_{1}^{i_{1}} \cdots x_{j}^{i_{j}-1} \cdots x_{k}^{i_{k}} .
$$

The notation $A_{x_{j}}$ is also used for the partial derivative $\partial A / \partial x_{j}$.

\section{B.2.4 Holomorphic functions and a law of permanence}

A complex-valued function $f(\mathbf{x})$ of several complex variables is holomorphic at $\mathbf{c}$ if it is continuous and differentiable in a neighborhood of $\mathbf{c}$. The notation $[\mathbf{a}, \mathbf{b}]$ is short for

$$
\left[a_{1}, b_{1}\right] \times \cdots \times\left[a_{k}, b_{k}\right] .
$$


Proposition 36 (A Law of Permanence for Functional Equations). Suppose

$$
A(\mathbf{x}), B(\mathbf{x}, y) \geq 0 \text {. }
$$

If there is an $\varepsilon>0$ such that

$$
A(\mathbf{x})=B(\mathbf{x}, A(\mathbf{x}))<\infty \quad \text { for } \mathbf{x} \in[\mathbf{0}, \varepsilon]
$$

then

$$
A(\mathbf{x})=B(\mathbf{x}, A(\mathbf{x})) \quad \text { for } \mathbf{x} \in[\mathbf{0}, \infty] .
$$

If furthermore $\mathbf{a}>\mathbf{0}$ and $A(\mathbf{a})<\infty$ then

$$
A(\mathbf{x})=B(\mathbf{x}, A(\mathbf{x})) \quad \text { for }\left|x_{i}\right| \leq a_{i}, 1 \leq i \leq k
$$

and $A(\mathbf{x})$ is holomorphic for $\left|x_{i}\right|<a_{i}, 1 \leq i \leq k$.

Proof. This is a special case of Hille's law of permanence for functional equations given in $\S 10.7$ of Vol. 2, [11].

\section{B.3 The Perron-Frobenius theory of nonnegative matrices}

The key to the main results of this paper are some simple observations based on the wellknown Perron-Frobenius theory of nonnegative matrices that was developed ca. 1910.

Proposition 37. Let $M$ be a nonnegative nonzero $k \times k$ matrix with real entries.

(a) $M$ has a real eigenvalue.

(b) The largest real eigenvalue $\Lambda(M)$ is positive and is given by

$$
\Lambda(M)=\max _{\mathbf{x}>0} \min _{1 \leq i \leq k} \frac{(M \mathbf{x})_{i}}{x_{i}} .
$$

(c) $\Lambda(M)$ is a simple root of the characteristic polynomial $p_{M}(\lambda)=\operatorname{det}(\lambda I-M)$.

(d) The eigenspace belonging to $\Lambda(M)$ is 1-dimensional, generated by a unique positive normalized eigenvector $\mathbf{v}_{M}$. (Normalized means the sum of the entries is 1).

Proof. (See $\S 2$ of Gantmacher [10].)

Note that Proposition 37(b) implies that for some $\mathbf{x}>\mathbf{0}$ one has $\Lambda(M)$ equal to $\min _{1 \leq i \leq k} \frac{(M \mathbf{x})_{i}}{x_{i}}$.

\section{Corollary 38.}

(a) A positive $k \times k$ matrix $M, k \geq 2$, has all diagonal entries $<\Lambda(M)$. 
(b) $\Lambda(X)$ is a nondecreasing function on the set of nonnegative matrices, that is, $M_{1} \leq$ $M_{2}$ implies $\Lambda\left(M_{1}\right) \leq \Lambda\left(M_{2}\right)$. Furthermore if every row [column] sum of $M_{1}$ is less than the corresponding row [column] sum of $M_{2}$ then $\Lambda\left(M_{1}\right)<\Lambda\left(M_{2}\right)$.

(c) $\Lambda(X)$ is a continuous function on the set of nonnegative matrices, where the matrices are thought of as points in $k^{2}$-space.

Proof. (Exercise.)

(Note: A special case of item (c) is stated on p. 2103 of Lalley [12], for certain Jacobian matrices denoted $J_{z}$, evaluated along certain curves.)

\section{References}

[1] Edward A. Bender, Asymptotic methods in enumeration. SIAM Rev. 16 (1974), 485515.

[2] Jason P. Bell, Stanley N. Burris, and Karen A. Yeats, Counting Rooted Trees: The Universal Law $t(n) \sim C \cdot \rho^{-n} \cdot n^{-3 / 2}$. The Electron. J. Combin. 13 (2006), R63 [64pp.]

[3] Jason P. Bell, Stanley N. Burris, and Karen A. Yeats, Characteristic Points of Recursive Systems. Preprint 2009, http://arxiv.org/abs/0905.2585v1

[4] E. Rodney Canfield, Remarks on an asymptotic method in combinatorics. J. Combin. Theory Ser. A 37 (1984), no. 3, 348-352.

[5] A. Cayley, Researches on the partition of numbers. Phil. Trans. Roy. Soc. London 146 (1856), 127-140.

[6] A. Cayley, On the theory of the analytical forms called trees. Phil. Magazine 13 (1857), 172-176.

[7] Michael Drmota, Systems of functional equations. Random Structures and Algorithms 10 (1997), 103-124.

[8] Michael Drmota, Random Trees. Springer, 2009.

[9] Philippe Flajolet and Robert Sedgewick, Analytic Combinatorics. Cambridge University Press, 2009.

[10] F.R. Gantmacher, Applications of the Theory of Matrices. Interscience Publishers, Inc., New York, 1959.

[11] E. Hille, Analytic function theory, Blaisdell Publishing Company, Waltham, 1962, 2 Volumes.

[12] Stephen P. Lalley, Finite range random walk on free groups and homogeneous trees. The Annals of Probability 21, No. 4 (1993), 2087-2130.

[13] A. Meir and J. W. Moon, On the altitude of nodes in random trees, Canadian Journal of Mathematics 30 (1978), 9971015.

[14] A. Meir and J.W. Moon, Some asymptotic results useful in enumeration problems. Aequationes Math. 33 (1987), 260-268. 
[15] A. Meir and J.W. Moon, On an asymptotic method in enumeration. J. Combin. Theory Ser. A 51 (1989), no. 1, 77 Erratum: J. Combin. Theory Ser. A 52 (1989), no. $1,163$.

[16] A.M. Odlyzko, Asymptotic enumeration methods. Handbook of Combinatorics, Vol. 1, 2, 1063-1229, Elsevier, Amsterdam, 1995.

[17] R. Otter, The number of trees. Annals of Mathematics 49 (1948), 583-599.

[18] G. Pólya and R.C. Read, Combinatorial enumeration of groups, graphs and chemical compounds. Springer Verlag, New York, 1987.

[19] J.J. Sylvester, On the partition of numbers. Quarterly J. Math. 1, (1855), 141-152.

[20] Alan R. Woods, Coloring rules for finite trees, probabilities of monadic second order sentences. Random Structures Algorithms 10 (1997), 453485. 


\section{Corrigendum - submitted May 13, 2011}

- page 11 , Lemma 7 requires the extra hypothesis " $\Sigma^{*}$ is well-conditioned".

- page 7 , line before the display giving $\Sigma^{(\alpha)}$ : change "a new variable $\alpha y_{0}$ " to "a new variable $y_{0} "$.

- page 9 , change $(x, \mathbf{y})$ to $(a, \mathbf{b})$.

- All references to Lemma 5(e) should be to Lemma 5 (c) and (d). Specifically: on page 9 drop the third paragraph from the bottom, change (d) to (c) in the next paragraph and change (e) to (d) in the last paragraph; on page 14 in the proof of Lemma 12 replace (e) by (d), and in the proof of Lemma 13 replace (e) by "(c) and (d)" .

- page 18, in the proof of Proposition $18 a=\rho$ should be moved to case (II).

- page 32, Proposition 37: "Let M be an irreducible nonnegative...". 